\title{
Improving recovery of member genomes from enrichment reactor microbial communities using MinION-based long read metagenomics
}

\author{
$4 \quad$ Krithika Arumugam ${ }^{1, *}$, Irina Bessarab ${ }^{2,}$, Xianghui Liu ${ }^{1}$, Gayathri Natarajan ${ }^{1}$, \\ 5 Daniela I. Drautz-Moses ${ }^{1}$, Stefan Wuertz ${ }^{1,3}$, Federico M. Lauro ${ }^{1,4}$, Ying Yu Law ${ }^{1}$, \\ 6




\begin{abstract}
New long read sequencing technologies offer huge potential for effective recovery of complete, closed genomes. While much progress has been made on cultured isolates, the ability of these methods to recover genomes of member taxa in complex microbial communities is less clear. Here we examine the ability of long read data to recover genomes from enrichment reactor metagenomes. Such modified communities offer a moderate level of complexity compared to the source communities and so are realistic, yet tractable, systems to use for this problem. We sampled an enrichment bioreactor designed to target anaerobic ammonium-oxidising bacteria (AnAOB) and sequenced genomic DNA using both short read (Illumina $301 \mathrm{bp} \mathrm{PE}$ ) and long read data (MinION Mk1B) from the same extraction aliquot. The community contained 23 members, of which 16 had genome bins defined from an assembly of the short read data. Two distinct AnAOB species from genus Candidatus Brocadia were present and had complete genomes, of which one was the most abundant member species in the community. We can recover a $4 \mathrm{Mb}$ genome, in 2 contigs, of long read assembled sequence that is unambiguously associated with the most abundant AnAOB member genome. We conclude that obtaining near closed, complete genomes of members of low-medium microbial communities using MinION long read sequence is feasible.
\end{abstract}

The development of long read sequencing technologies, such as the Oxford Nanopore Technology MinION and Pacific Biosciences SMRT offer huge potential for effective recovery of complete, closed genomes $[1,2]$. While much progress has been made towards this objective for single species isolates $[3,4]$, the ability of these methods to recover genomes of member taxa in complex microbial communities (microbiomes) is less clear.

To date there is limited long read data available in the metagenome context. Several recent studies have used MinION or PacBio sequencing on highly enriched reactor communities, for example a recent study using PacBio long read sequencing to recover a complete, closed genome of Kuenenia stuttgartiensis which was present at around $95 \%$ relative abundance [5]. Other studies have obtained long read data or combined long and short read data on more complex communities, for example from moderately enriched bioreactor communities [6], co-culture enrichments [7], marine holobionts [8] or from full scale anaerobic digester communities [9]. There are also several datasets which provide long and short read data from mock communities $[10,11,12]$, and which constitute important new benchmarking data. New long read analysis methods [13] and binning algorithms designed for long read metagenome data [14] are becoming available, anticipating the future expansion of metagenome data from these new instruments.

Here we examine the ability of long read data to recover genomes from enrichment bioreactor metagenomes. Such modified communities offer a moderate level of complexity compared to the source communities and so are realistic, yet tractable, systems to use for this problem. Specifically, we sampled an enrichment bioreactor targeting anaerobic 
ammonium-oxidising bacteria (AnAOB), extracting genomic DNA and obtaining both short read (Illumina MiSeq 301bp PE) and long read data (MinION Mk1B) from the same aliquot. We perform a comparative analysis of different long read assemblers and assembly workflows, using the draft genomes extracted from the short read assembly as an independent references to assess the ability of long read assemblies to capture genome of member species of the community. Our findings show that the long read metagenomics can recover a complete, near-closed genome of the most abundant community member, even in the presence of multiple species of the same genus.

\section{Methods}

\section{Overall approach}

We studied a bioreactor community established to enrich for anaerobic ammonia-oxidizing bacteria (AnAOB), all known species of which belong to the phylum Planctomycetes. Previously we used this enrichment reactor community to recover a draft genome of a member of the genus Candidatus Brocadia [15]. We sampled the suspended biomass of the reactor, extracted DNA and performed both Illumina and Nanopore DNA sequencing from the same extracted aliquot. Such enrichment reactor communities have the advantage of being only moderately complex relative to their source communities and therefore provide an ideal tradeoff between realistic complexity and tractability in relation to member genome recovery.

We constructed long read assemblies and then used draft genomes obtained from short read assemblies for the purpose of assessment. This approach takes advantage of the fact of our having obtained data from both sequencing modalities using the same DNA aliquot, and leverages current understanding of short read metagenome assembly binning and quality assessment procedures $[16,17]$. We tested five different long read assemblers or assembly workflows, namely Canu [18], Miniasm [19], SMARTDenovo [20], Spectrassembler [21] and Unicycler [22]. We note that while these softwares are not designed or endorsed for metagenome analysis, our experimental design explicitly tested their ability to be used in this setting.

\section{Enrichment bioreactor and sampling}

As previously described [15], we (Y.Y.L, G.N, S.W) developed a protocol to enrich for AnAOB organisms using a sequencing batch reactor seeded with return activated sludge from a water reclamation plant (Public Utilities Board, Singapore). The reactor was fed synthetic wastewater containing ammonium and nitrite and operated at $35^{\circ} \mathrm{C}$. The reactor showed a characteristic reddish-brown biofilm on the walls of the reactor (characteristic of AnAOB bacteria), and we observed simultaneous ammonium and nitrite depletion consistent with the occurrence of Anammox. We confirmed the presence of AnAOB species 
within the community using FISH, 16S amplicon sequencing and shotgun metagenomics [15]. At the time of sampling for the present study (10/2016) the reactor community had been established for approximately 9 months.

\section{DNA extraction}

DNA was extracted from sampled biomass with the FastDNA ${ }^{\mathrm{TM}}$ SPIN kit (MP Biomedicals) for Soil, using using $4 \times$ bead beating with a FastPrep homogeniser (MP Biomedicals) followed by cleanup with Genomic DNA Clean \& Concentrator ${ }^{\text {TM }}-10$ (Zymo Research).

\section{Short read sequencing}

Genomic DNA Library preparation was performed using a modified version of the Illumina TruSeq DNA Sample Preparation protocol. We then performed a MiSeq sequencing run with a read length of $301 \mathrm{bp}$ (paired-end). The raw short read data is publicly available from the NCBI via BioProject accession PRJNA471614 or Sequence Read Archive accession SRR8168439.

\section{Long read sequencing}

Library preparation was constructed from $1.7 \mu \mathrm{g}$ of genomic DNA using the SQK-LSK 108 Ligation Sequencing Kit (Oxford Nanopore Technologies). Sequencing was performed on a MinION Mk1B instrument (Oxford Nanopore Technologies) using a SpotON FLO MIN106 flowcell (FAF05082) and R 9.4 chemistry. Data acquisition was performed using MinKNOW version 1.3.30 running on a HP ProDesk 600G2 computer (64-bit, 16Gb RAM, $2 \mathrm{~Tb}$ SSD HD) running Windows10. The raw long read data is publicly available from the NCBI via BioProject accession PRJNA471614 or and Sequence Read Archive accession SRR7169548.

\section{Analysis of short read sequence data}

The raw FASTQ files were processed using cutadapt (version 1.14) [25] using the following arguments: --overlap 10 -m 30 -q 20,20 --quality-base 33. We initially characterised the community data using Ribotagger [26] selecting the V4 region and annotating ribotags to the SILVA database [27]. We calculated relative abundance of each ribotag by dividing their reads counts by the total read count from all ribotags. Single-sample metagenome assembly of the short read sequence was constructed using Newbler v2.9. The contigs generated from short read data are hereafter denoted as short read assembled contigs (SRAC). We identified 16S genes within contigs using the --search16 module of USEARCH (version 10.0.240, 64 bit) [28], and annotated them using the SILVA::SINA webserver (using default parameters) [29]. Genes (open reading frames) were predicted from all contigs using MetaGeneMark [30] and annotated to RefSeq NR (January 2016 version) 
[24] using DIAMOND v0.8.22.84 (running default parameters) [31]. We identified putative member genomes using MetaBAT [32]. For each identified bin we performed taxonomic level analysis using CheckM [33] and by applying the $K$-statistic methods of Williams et al. [34].

\section{Analysis of long read sequence data}

Basecalling was performed using Albacore version 2.2.4. Adaptor trimming was performed using Porechop [35] with default settings. We performed taxonomic analysis of long read data using MEGAN-LR [13]. We assembled long read data using each of the following long read assemblers: Canu [18], Miniasm [19], SMARTDenovo [20], Spectrassembler [21] and Unicycler [22], using default setting unless otherwise stated. In Canu there is a required parameter (genomeSize) that sets the expected genome length, and which controls the number of reads used by assembler. Because this estimate is essentially meaningless in the metagenome context, we computed multiple assemblies using the following values of genomeSize: $3.4 \mathrm{Mbp}, 5 \mathrm{Mbp}, 12 \mathrm{Mbp}, 25 \mathrm{Mbp}, 50 \mathrm{Mbp}$ and $120 \mathrm{Mbp}$. Contigs generated from long read data are hereafter denoted as long read assembled contigs (LRAC). The number of reads used in each assembly was estimated by mapping long read to LRAC sequence with minimap2 [36] and using samtools-1.6 to calculate the number of aligned reads [37].

\section{Comparative analysis of long and short read assemblies}

We used BLASTN (version 2.4.0+) [23] to examine the degree of sequence alignment between LRAC and SRAC sequences. We treated the LRAC as the subject sequences and the SRAC as the query sequences, using default BLASTN parameters and without the results outputted in tabular format. To assess the extent to which SRAC sequence aligned to LRAC sequence, we computed ratio of the alignment length to the length of the query (SRAC) sequence $(a l 2 q l)$.

\section{Results and Discussion}

\section{Community profiling and member genome recovery using short read data}

Community profiling using RiboTagger [2] detected a total of 23 member taxa (defined by $16 \mathrm{~S} \mathrm{~V} 4$ region sequence captured in metagenome reads). Of these 7 taxa showed a relative abundance of at least 5\% (Fig. 1A). In order of decreasing relative abundance, those seven taxa are Candidatus Brocadia (genus), a member of phylum Armatimonadetes, an unknown taxa (no kingdom assigned), a member of genus Denitratisoma, a second $C a$. Brocadia (genus) taxon, a member of family Anaerolineaceae and another unknown taxon. The short read data was subsequently assembled (Table S1) and automatically binned (see 
Methods and Fig. 1B), which resulted in 16 bins being defined, of which 4 demonstrated an estimated completeness of $>95 \%$ and contamination $<5 \%$ (Table S2). Taxonomic analysis of each bin was based on presence of $16 \mathrm{~S}$ sequence, marker gene analysis [33] and analysis of ORF-level annotations [34]. The results were qualitatively consistent with the $16 \mathrm{~S}$ analysis, and two species of $C a$. Brocadia identified (bin 2 and bin 6, with mean base coverage of 150 and 31, respectively), a member of phylum Armatimonadetes (bin 1, mean base coverage 180) and a member of phylum Chloroflexi (bin 7, mean base coverage, 150) (Fig. 1B, Table S2-S3). We observed that bin 3, comprised of a set of 26 contigs (approximately $201 \mathrm{k}$ bp of sequence, with mean base coverage 142) that are strongly associated with $\mathrm{Ca}$. Brocadia (Table S2-S3), overlapped in the coverage-GC plane with bin 2 and we therefore subsequently combined those two bins under the assumption that they were spuriously separated by the binning procedure (referred to hereafter as bin 2).

\section{Nanopore sequencing and initial taxonomic analysis of long read data}

The MinION run generated a total of 115,383 basecalled reads, of which 95,571 held a quality score $>7$ (Table 1) and $93.4 \%$ were greater than $500 \mathrm{bp}$ in length. The mean read length was 4,677 bp with the longest read being $112,729 \mathrm{bp}$. There were 6,324 reads with a length greater than $10,000 \mathrm{bp}$. A total of 539,410,340 bp of sequence was generated from the run.

Taxonomic assignments using MEGAN-LR were obtained for 88,097 reads $(76.4 \%$ of all reads), representing a total of $326 \mathrm{M}$ aligned bases, of which $291 \mathrm{Mbp}$ were annotated to bacterial phyla. The most prominent phyla as ranked by aligned base length was Planctomycetes $(157 \mathrm{Mb}$ or $54 \%$ of total sequence aligned to phylum level), Proteobacteria (69.7Mb, 24\%), Chloroflexi (21.0 Mbp) and Armatimonadetes (1.79 Mbp, 6.14\%) (Table S4). A total of $162 \mathrm{Mbp}$ of alignments were obtained at genus level, with $93.4 \%$ of these attributable to $\mathrm{Ca}$. Brocadia. Around $2 \%$ of aligned bases were attributable to genus Nitrospira, with the remaining detected genera each accounting for less than $1 \%$ of aligned bases (Table S5).

\section{Long read assembly}

Using the long read data, we constructed assemblies using five assemblers or assembly workflows (Table 2). The total amount of assembled sequence varied between approximately $4.8 \mathrm{Mb}$ (Canu with genomeSize parameter set to $3.4 \mathrm{Mbp}$ ) and $11.2 \mathrm{Mbp}$ (Canu with genomeSize parameter set to $50 \mathrm{Mbp}$ or $120 \mathrm{Mbp}$ ) (Table 2). Examination of the GC-length data showed a consistent tendency for longest contig to be clustered around a GC of approximately 0.4, consistent with the high abundance of AnAOB (Fig. 2). The distribution of LRAC sequence length varied across assemblers (Fig. 2), but all assemblers returned at least one LRAC sequence greater than $1 \mathrm{Mb}$ in length, with Canu and SMARTdenovo returning 2 sequences longer than $1 \mathrm{Mb}$. Between $33 \%$ and $45 \%$ of long reads 
contributed to LRAC sequence, dependent on what assembler or assembly workflow was used (Table 2).

\section{Taxonomic and genomic analysis of long read assembled sequence}

To begin taxomomic analysis of LRAC sequence, we next examined whether $16 \mathrm{~S}$ gene sequences were present using homology search, and if so, obtained their taxonomic annotations. To do so we aligned all full length 16S genes identified in the short read assembly to all LRAC sequences. In the short read assembly, we identified a full length $16 \mathrm{~S}$ gene in bin 2 (residing on contig00593) that was aligned to SILVA annotated sequence from Ca. Brocadia with $95 \%$ identity. That sequence mapped with over $99 \%$ identity to LRAC sequences generated by Canu and Unicycler and close to $99 \%$ identity in the case of Spectrassembler. In the other two assemblers, the degree of alignment were lower at $88 \%$ and $73 \%$ for Miniasm and SMARTdenovo, respectively. A second full length 16S gene annotated to $C a$. Brocadia in SILVA and recovered from bin 6 was aligned to the same LRAC locations as the bin 2 sequence, but with lower percent identity in all instances (alignment statistics for these two 16S sequences are provided in Supplementary Datafile 1).

We next examined whether these LRAC sequences constituted complete or near-complete genomes of member species. Using BLASTN, we aligned SRAC sequences (treated as queries) to the LRAC sequences (treated as subject) and then categorised the homology search statistics by the bin of origin of the aligned SRAC sequence. We summarised the bulk alignment statistics for each LRAC partitioned by SRAC-bin using the mean of the median of the distribution of the percent identity (pident) and the median of the distribution of the alignment length to query (SRAC) length (al2ql; expressed as a percentage to be on the same scale as pident) (Fig. 3). With the exception of Spectrassembler the longest LRAC sequences showed a strong degree of alignment with SRAC sequences arising from the highest abundance $C a$. Brocadia bin (bin 2) and to a lesser degree with SRAC sequences from the lower abundance $C a$. Brocadia taxon (bin 6). No other LRAC sequence demonstrated a high degree of association with any other genome bin defined in our analysis. We therefore supposed that these LRAC sequences capture substantial sized fragments of the most abundant $C a$. Brocadia genome present in the community, and then developed further analyses to test this notion. We now focused attention of the Canu assembly generated using genomeSize $=3.4 \mathrm{Mb}$, as this gave the longest single LRAC sequence from any workflow. This LRAC sequence (hereafter referred to as tig00000001) contained the full length $16 \mathrm{~S}$ referred to above. Given this sequence also has a mean GC close to 0.4, we can consider it a candidate for being a large genome fragment of Ca. Brocadia present in the reactor community. This contig had a mean long read base coverage of 48 (s.d: 12) (Fig. S1).

If this LRAC sequence represents large genomic fragments from a member species of the community, then we would predict the SRAC sequences from the cognate short read assembly bin will align in a spatially uniform fashion across it. We therefore examined the 
spatial distribution of SRAC alignments across the entire the cohort of LRAC assembly using dot plots, generating these for the following sets of SRAC sequence 1) all contigs that are members of neither bin 2 nor bin 6 ;2) contigs that are members of bin 6 and 3) contigs that are members of bin 2 . We further subset for those alignments that were full length (defined as alignments exhibiting al2ql>95\%). The results of each of the corresponding six analyses are shown in Figs 4-9. Each plot shows a set of four panels, the left most of which is a dot plot showing the alignments of the SRAC sequence on the entire LRAC assembly, the next rightmost panels showing the corresponding BLAST statistics for SRAC alignments, namely al $2 q l$ and pident, and the rightmost panel shows the length of the aligned SRAC sequences.

Starting with the SRAC sequences that are neither members of bin 2 or bin 6 , we observe only a small number of alignments to tig00000001 with low values of al2ql (Fig. 4), and four alignments of full length, high percent identity to other short LRAC sequences in the assembly (Fig. 5). SRAC sequences from each of the two $\mathrm{Ca}$. Brocadia bins showed markedly different patterns of alignment. Only SRAC sequences from bin 2 showed spatially uniform tiling across tig00000001 (Fig. 8), with a high degree of alignment and percent identity (Fig. 9). SRAC sequences from bin 6 also showing a large number of alignments, but at greatly reduced number when we considered only full length alignments (Fig. 7). Based on this analysis, we can conclude that tig00000001 appears to derive from genome fragments associated with the $C a$. Brocadia species captured in bin 2, but not bin 6 , in the short read assembly. We note that the reduced degree of percent identity from SRAC sequences from bin 6 is not a consequence low coverage (leading to a systematic reduction in contig length), as a systematic relationship between SRAC length and coverage is only evident at coverage values $<10$, not at the coverage ranges observed for either $C a$. Brocadia bin (Fig. S2A).

We observed that the second longest contig in the assembly, hereafter referred to as tig00000002 (length: $362 \mathrm{k}$ bp; mean base coverage of 42, s.d: 12, see Fig. S2B), showed a similar pattern of alignments from the bin 2 SRAC sequence, suggesting that it is another, shorter fragment of the $C a$. Brocadia captured in tig00000001. The lack of repetition of SRAC alignments relative to those aligned to tig00000001 suggests this is a distinct genome fragment, not a shorter sub-fragment of the tig00000001 sequence.

\section{Refining short read bin composition reduces alignment gaps in long read assembly}

Despite the spatially uniform tiling of SRAC sequences from bin 2 sequences across the long read assembly, the dot plots suggest the presence of a number of gaps where no alignments exist. We quantified the extent of this and observed that $21.3 \%$ and $44.6 \%$ of tig00000001 and tig00000002 are not covered by bin 2 SRAC alignments, respectively. Examination of the default bin assignments from MetaBAT (Fig. 1) suggest both bin 2 and bin 6 contain a proportion of outliers, which are be unlikely to derive from their 
cognate genomes. Because neither the SRAC bin-genomes nor the LRAC sequences can be considered a gold-standard assembly of given member species, we refined the short assembly bin membership and re-examined the extent of tiling across the cognate LRAC sequence, to determine if the occurrence of alignment gaps was determined largely by bin membership, incomplete short read assembly and/or subsequences in the long read assembly associated with sequencing or assembly errors. To expand the total amount of candidate sequence derived from the bin $2 \mathrm{Ca}$. Brocadia genome we included all SRAC contigs at least $1 \mathrm{kbp}$ in length and performed the following procedure. We started with the bin 2 contig that contained the full length 16S sequence (contig00593), and extracted contig sets defined by the $k$-nearest neighbours of that contig in the binning plane. We grew these neighbourhoods in increments of 10 contigs, starting with 10 contigs and finishing with 550 contigs (Fig. 10A). For each of these neighbourhoods, we calculated 1) CheckM genome quality statistics (Fig. 10B); 2) total sequence length (Fig. 10C); 3) ORF-level annotation statistics summarised at neighbourhood level for family and genus level for $C a$. Brocadiaceae (Fig. 10D) and Ca. Brocadia (Fig. 10E), respectively; 4) the proportion of LRAC sequence of tig00000001 and tig00000002 that contain no aligned SRAC sequence from bin 2 (Fig. 10F); 4) the number of bin 2 SRAC sequenced that were unaligned to LRAC sequences (Fig. 10G). When calculating gaps in the alignments to LRAC sequence, we only considered full length SRAC aligned sequences (i.e. those with al2ql>0.95).

Examining this contig neighbourhood analysis, we observe that $90 \%$ completeness as defined by CheckM is attained after the $k=230$ neighbourhood, with non-zero values of contamination and strain heterogeneity appearing at $k=240$ and $k=440$, respectively. We note that contamination never exceeds $3.85 \%$ which is below the $5 \%$ threshold considered to be a high quality draft metagenome assembled genome [17]. Visually, the occurrence of contamination is associated with neighbourhoods starting to impinge on the region of the binning plane associated with contigs in bin 6 , as well as outlier contigs from other taxon bins. Total sequence length increased in a near-linear fashion until $k=220$, attaining a length of $2.67 \mathrm{Mb}$, after which a more gradual increase in length was observed. High values of the $K$-statistic were observed across all neighbourhoods (with minimal signal from other taxa), with a slight decrease towards higher values of $k$. Interestingly, for the $k=230$ neighbourhood, there remains $36 \%$ and $52 \%$ of tig00000001 and tig00000002 that contain no aligned, full-length SRAC sequence. By $k=400$ these proportions had dropped to $15 \%$ and $33 \%$ respectively, with their nadir values of $10 \%$ and $27 \%$ being observed at $k=530$ and $k=520$, respectively. We also note that all SRAC sequences from the neighbourhood are aligned to an LRAC sequence until the $k=320$ neighbourhood, and across all neighbourhoods a total of 5 SRAC sequences never align to either LRAC sequence. The combination of contamination and strain heterogeneity statistics, along with the appearance of contigs that do not map to LRAC sequences, suggest that the short read derived genome is most complete and uncontaminated between the $k=310$ and $k=430$ neighbourhoods, which at the latter contains a total of $3.6 \mathrm{Mb}$ of sequence.

Collectively these observations suggest that as defined by LRAC sequences, the AnAOB 
genome arising from bin 2 of the short read assembly, is just over $4 \mathrm{Mb}$ in length and is captured by one long contig (tig00000001 and one shorter contig (tig00000002) of lengths $3,698,683 \mathrm{bp}$ and $362,151 \mathrm{bp}$, respectively. The gaps in the coverage of SRAC sequences onto these LRAC draft genome is consistent with incomplete assembly of cognate genome using the short read approach. The estimated length of $4 \mathrm{Mb}$ is consistent with previous AnAOB genome estimates, including a recent complete, closed assembly of Kuenenia stuttgartiensis using PacBio sequencing [5]. Previous short read assemblies, obtained from metagenome survey (MAG), are generally shorter than the estimated length by up to $1 \mathrm{Mb}$, consistent with the above findings.

\section{Summary and Conclusions}

In this paper we examine the capacity of long read data, generated by a Nanopore MinION sequencer, to capture member genomes of a microbial community of low-medium complexity. We show that we can recover a complete, near closed genome of the most abundant community member, specifically an anaerobic ammonium oxidising bacterium (AnAOB) belonging to genus $\mathrm{Ca}$. Brocadia. We also show that we can unambiguously resolve the genome of this species without contamination from a lower abundance community member of the same genus. Our results also cast light on the limitations of short read metagenome assembly, and the general challenges associated with accurate recovery of member genomes from complex microbial communities.

Long read metagenomics will likely represent an area of substantive activity over the next several years, particularly in regard to the recovery of complete genomes from member species. Despite great progress in the last decade in developing procedures to obtain short read metagenome-assembled genomes (MAG) [16, 17], substantial limitations of these approaches have become evident, including problems related to the use of multi-sample co-assemblies [38, 39], the challenges of resolving genomes to strain level [40], and the challenges of extracting MAGs from communities of moderate to high ecological complexity $[41,42,43]$. Accordingly, several recent papers have combined short read with emerging complementary techniques such as HiC metagenomics [42, 44], and it is likely to that the combination of short and long read metagenomics will also yield considerable progress in defining higher quality MAGs than is possible using short read metagenome assembly procedures alone. In the present paper, we seek to establish the feasibility of such hybrid long-short metagenomic approaches, starting with communities of low-moderate complexity.

Our analysis is based on the use of a single DNA aliquot co-assayed with both long and short sequencing, thus avoiding the introduction of any difference between the two sets of sequence that could be attributed to ecogenomic differences. Our analysis proceeds on the basis that neither SRAC nor LRAC sequence is known to provide a true reference genome, and so we seek to understand and characterise the degree of agreement between them 
[45]. Although error prone MinION sequence can be corrected using higher quality short read sequences, in this case, we have deliberately kept the two sources of data separate so as to not introduce any positive bias in our SRAC to LRAC alignment analysis. One consequence of this choice is that direct use of the LRAC assembly is limited in relation to prediction of genes (open reading frames), due to the greatly increased frame-shift rate in coding sequence [13]. Thus in this setting, we would consider our LRAC assembly to be more akin to a genome-wide scaffold for higher quality short read genome fragments, rather than an assembly per se. We note that we and others are actively working on correction procedures for frame-shift errors at the time of writing, however, the issues of high error rates in MinION sequence remains a substantial weakness of this technology.

We observe that most of the extant long read assembler or assembly workflows provide broadly comparable results in relation to contig generation (Fig. 2 and Table 2) and recruitment of bin-specific SRAC sequence (Fig. 3), although we note that some behaviours are counter-intuitive. For example, in using Canu, increasing the genomeSize parameter to $\geq 5 \mathrm{Mb}$ results in splitting of the large contig observed in the $3.4 \mathrm{Mb}$ version, despite the increased amount of sequence being assembled. We also note that less than $50 \%$ of long reads are typically used in the assembly, for reasons that are unclear. Thus, further development of metagenome assemblers and assembly workloads tailored for long read data, or hybrid, short-long read data, is clearly needed. These data were obtained during a phase of rapid development of MinION kits and protocols, only generating under 1Gb of sequence from these complex environmental samples, and we anticipate more comprehensive results using more recent datasets, given the improvement in yield, read length and quality now available from the same technology.

The concept of genome completeness remains problematic in MAG-based analyses of shotgun metagenome data. Current methods for estimating genome completeness from short read data are based on marker gene sets whose membership is defined by the fact of having a single genomic copy. Typically, these are of order of around 100 genes, and by definition such sets cannot accurately estimate the completeness of a genome. In the present case, we see that high level completeness in the SRAC sequence are occurring at total sequence length that is around $1.4 \mathrm{Mb}$ less than the genome length inferred from LRAC based analyses. In fact we would argue that given the complexity of how short read contigs from many member species will be distributed in a given binning space, there must remain a fundamental ambiguity in resolving genome content using these approaches, as highlighted by our neighbourhood based reanalysis of bin 2 . While the backbone of the genome is certainly captured under these criteria, knowing when one has accurately captured a whole genome remains challenging and reinforces the view that MAG-based analysis can likely only resolve a working model of the genome (or pangenome) of a given community member in most circumstances. In the present study we have also used completely automated methods, with no recourse to manual or subjective decision making that appear to be commonly deployed in the refinement of MAG draft genomes and represent a challenge to the conduct of reproducible research in this area. 
Further development of long read metagenomics may in fact obviate the need for complex binning procedures, with methods refocusing on the evaluation of LRAC sequences as putative draft genomes. Either way, it would appear as if conduct of hybrid shortlong read metagenome surveys will be essential for resolving these challenges for the foreseeable future. The engagement of other complementary approaches, namely single cell metagenomics [46], HiC-metagenomics [44] or FACS-generated mini-metagenomes [43] in combination with MAG approaches, will likely permit substantial progress.

\section{List of Supplementary Materials}

Supplementary items appear with this document unless otherwise stated.

- Table S1: Summary statistics for the short read assembly

- Table S2: CheckM statistics for short read assembly bins

- Table S3: $K$-statistics for short read assembly bins at phylum, family and genera levels

- Table S4: Summary of phyla-level analysis of long read data using MEGAN-LR

- Table S5: Summary of genera-level analysis of long read data using MEGAN-LR

- Supplementary Datafile 1: Alignment statistics for SRAC derived 16S (xlsx file)

- Figure S1: Per-base long read coverage for AnAOB-associated LRAC sequences

- Figure S2: Relationship between length and coverage in the short-read metagenome assembly

\section{Author contributions}

The study was conceptualised by RBHW and designed by RBHW and IB. GN, YYL and SW setup and operated enrichment reactors, and obtained samples with IB. IB performed DNA extractions and performed long read sequencing, with IB and FL designed long read sequencing experiments. DID performed short read sequencing. KA, RBHW, XHL and DHH designed and/or performed data analysis. All authors contributed to data interpretation. RBHW wrote the manuscript with specific contributions from all other authors.

\section{Acknowledgements}

This research was supported by the Singapore National Research Foundation and Ministry of Education under the Research Centre of Excellence Programme, and by program grant 
1301-IRIS-59 from the National Research Foundation (NRF). We thank Gavin Huttley (Australian National University) for critical feedback on sequence analysis. Early version of this work were presented at APBC2018 (Yokohama, Japan, January 15-17 2018) and ISME17 (Leipzig, Germany, August 12-17 2018)

\section{References}

[1] Loman, N.J., Quick, J., Simpson, J.T. (2015) A complete bacterial genome assembled de novo using only Nanopore sequencing data. Nat. Methods 12 (8): 733-5

[2] Wick, R.R., Judd, L.M., Gorrie, C.L., Holt, K.E. (2017). Completing bacterial genome assemblies with multiplex MinION sequencing, Microb. Genom. 3(10): e000132

[3] Doyle, L.E., Williams, R.B.H., Rice, S.A., Marsili, E., Lauro, F.M. (2018). Draft genome sequence of Enterobacter sp. Strain EA-1, an electrochemically active microorganism isolated from tropical sediment, Genome Announcements 6(9): e00111-18.

[4] Daebeler, A., Herbold C.W., Vierheilig, J., Sedlacek C.J., Pjevac, P., Albertsen, M., Kirkegaard, R.H., de la Torre, J.R., Daims, H., Wagner, M. (2018). Cultivation and genomic analysis of "Candidatus Nitrosocaldus islandicus", an obligately thermophilic, ammonia-oxidizing Thaumarchaeon from a hot spring biofilm in Graendalur Valley, Iceland. Frontiers in Microbiology 9: 193.

[5] Frank, J., Lücker, S., Vossen, R.H.A.M., Jetten, M.S.M., Hall, R.J., Op den Camp, H.J.M., Anvar, S.Y. (2018). Resolving the complete genome of Kuenenia stuttgartiensis from a membrane bioreactor enrichment using Single-Molecule Real-Time sequencing. Scientific Reports. 8(1): 4580.

[6] Andersen, M.H., McIlroy, S.J., Nierychlo, M., Nielsen, P.H., Albertsen, M. (2018). Genomic insights into Candidatus Amarolinea aalborgensis gen. nov., sp. nov., associated with settleability problems in wastewater treatment plants, Systematic and Applied Microbiology, available online 16 August 2018 https://doi.org/10.1016/j.syapm.2018.08.001

[7] Driscoll, C.B., Otten T.G., Brown, N.B., Dreher, T.W. (2017). Towards long-read metagenomics: complete assembly of three novel genomes from bacteria dependent on a diazotrophic cyanobacterium in a freshwater lake co-culture, Standards in Genomic Sciences. 12: 9

[8] Slaby, B.M., Hackl, T., Horn, H., Bayer, K., Hentschel, U. (2017). Metagenomic binning of a marine sponge microbiome reveals unity in defense but metabolic specialization, ISME Journal, 11: 2465-2478. 
[9] Frank, J.A., Pan, Y., Tooming-Klunderud, A., Eijsink, V.G.H., McHardy, A.C., Nederbragt, A.J. (2016). Improved metagenome assemblies and taxonomic binning using long-read circular consensus sequence data, Scientific Reports, 6: 25373.

[10] Singer, E., Andreopoulos, B., Bowers, R.M., Lee, J., Deshpande, S., Chiniquy, J., Ciobanu, D., Klenk, H-P., Zane, M., Daum, C., Clum, A., Cheng, J-F., Copeland, A., Woyke, T. (2016) Next generation sequencing data of a defined microbial mock community. Scientific Data 3:160081.

[11] Brown, B.L., Watson, M., Minot, S.S., Rivera, M.C. Franklin, R.B. (2017). MinION nanopore sequencing of environmental metagenomes: a synthetic approach. GigaScience 6: 1-10.

[12] Nanopore GridION and PromethION Mock Microbial Community Data Community Release, Release 2 (2018-10-17). https://github.com/LomanLab/mockcommunity

[13] Huson, D.H., Albrecht, B., Bagci, C., Bessarab, I., Gorska, A., Jolic, D., Williams, R.B.H (2018). MEGAN-LR: New algorithms allow accurate binning and easy interactive exploration of metagenomic long reads and contigs, Biology Direct 13: 6

[14] Laczny, C.C., Kiefer, C., Galata, V., Fehlmann, T., Backes, C., Keller, A. (2017). BusyBee Web: metagenomic data analysis by bootstrapped supervised binning and annotation. Nucleic Acids Res. 45 (W1): W171-W179

[15] Liu, X.H., Arumugam, K., Natagaran, G., Seviour, T.W., Drautz-Moses, D.I., Wuertz, S., Law, Y.Y., Williams, R.B.H. (2018). Draft genome sequence of an "Candidatus Brocadia" bacterium enriched from tropical-climate activated sludge, Genome Announcements, in press. Preprint biorxiv.org/content/early/2017/04/24/123943

[16] Quince, C., Walker, A.W., Simpson, J.T., Loman, N.J., Segata, N. (2017), Shotgun metagenomics, from sampling to analysis, Nature Biotechnology 35: 833-84.

[17] Bowers, R.M., Kyrpides, N.C. Stepanauskas, R., Harmon-Smith, M., Doud, D. et al. (2017). Minimum information about a single amplified genome (MISAG) and a metagenome-assembled genome (MIMAG) of bacteria and archaea, Nature Biotechnology 35: 725-731.

[18] Koren, S., Walenz, B.P., Berlin, K., Miller, J.R., Bergman, N.H., Phillippy, A.M. (2017). Canu: scalable and accurate long-read assembly via adaptive $k$-mer weighting and repeat separation, Genome Research, 27(5): 722-736.

[19] Li, H. (2016). Minimap and miniasm: fast mapping and de novo assembly for noisy long sequences, Bioinformatics 32(14): 2103-2110.

[20] SMARTdenovo https://github.com/ruanjue/smartdenovo 
[21] Recanati, A., Brüls, T., d'Aspremont, A. (2017). A spectral algorithm for fast de novo layout of uncorrected long nanopore reads, Bioinformatics 33(20): 3188-3194.

[22] Wick, R.R., Judd, L.M., Gorrie, C.L. Holt, K.E. (2017). Unicycler: Resolving bacterial genome assemblies from short and long sequencing reads, PLoS Computational Biology 13(6): e1005595.

[23] Altschul, S.F., Gish, W., Miller, W., Myers, E.W., Lipman, D.J. (1990). Basic local alignment search tool. J. Mol. Biol. 215: 403-410.

[24] O'Leary NA, Wright MW, Brister JR, Ciufo S, Haddad D et al. (2016). Reference sequence (RefSeq) database at NCBI: current status, taxonomic expansion, and functional annotation. Nucleic Acids Res. 44(D1): D733-45

[25] Martin, M. (2011). Cutadapt removes adapter sequences from high-throughput sequencing reads. EMBnet.journal, 17(1): 10-12.

[26] Xie, C., Goi, W.C., Huson, D.H., Little, P.F.R., Williams, R.B.H. (2016). RiboTagger: fast and unbiased $16 \mathrm{~S} / 18 \mathrm{~S}$ profiling using whole community shotgun metagenomic or metatranscriptome surveys, BMC Bioinformatics 17(Suppl 19): 1378

[27] Pruesse, E., Quast, C., Knittel, K., Fuchs, B.M., Ludwig, W., Peplies, J,, Glckner, F.O. (2007). SILVA: a comprehensive online resource for quality checked and aligned ribosomal RNA sequence data compatible with ARB. Nucleic Acids Research 35: 7188 7196.

[28] Edgar, R.C. (2017). SEARCH_16S: A new algorithm for identifying $16 \mathrm{~S}$ ribosomal RNA genes in contigs and chromosomes. http://biorxiv.org/content/early/2017/04/04/124131

[29] Pruesse, E., Peplies, J., Glöckner, F.O. (2012) SINA: accurate high-throughput multiple sequence alignment of ribosomal RNA genes. Bioinformatics 28: 1823-1829

[30] Zhu, W., Lomsadze, A., Borodovsky, M. (2010). Ab initio gene identification in metagenomic sequences. Nucleic Acids Research 38: e132.

[31] Buchfink, B., Xie, C., Huson, D.H. (2015). Fast and sensitive protein alignment using DIAMOND, Nature Methods 12(1): 59-60.

[32] Kang, D.D., Froula, J., Egan, R., Wang, Z. (2015). MetaBAT, an efficient tool for accurately reconstructing single genomes from complex microbial communities, PeerJ, 3, e1165.

[33] Parks, D.H., Imelfort, M., Skennerton, C.T., Hugenholtz, P., Tyson, G.W. (2015). CheckM: assessing the quality of microbial genomes recovered from isolates, single cells, and metagenomes" Genome Research, 25, 1043-1055. 
[34] Williams, R.B.H., Liu, X.H., Arumugam, K. (2018). Annotation statistics for exploratory analysis of metagenome assembly binning data, unpublished manuscript.

[35] Porechop. https://github.com/rrwick/Porechop

[36] Li, H. (2018). Minimap2: pairwise alignment for nucleotide sequences. Bioinformatics 34 (18): 3094-3100.

[37] Li, H., Handsaker, B., Wysoker, A., Fennell, T., Ruan, J., Homer, N., Marth, G., Abecasis, G., Durbin, R.; 1000 Genome Project Data Processing Subgroup. The Sequence Alignment/Map format and SAMtools, Bioinformatics. 25(16): 2078-2079.

[38] Olm, M.R., Brown, C.T., Brooks, B., Banfield, J.F. (2018). dRep: a tool for fast and accurate genomic comparisons that enables improved genome recovery from metagenomes through de-replication, ISME J. 11(12): 2864-2868

[39] Miller, I.J., Rees, E.R., Ross, J., Miller, I., Baxa, J., Lopera, J., Kerby, R.L., Rey, F.E., Kwan, J.C. (2018). Autometa: Automated extraction of microbial genomes from individual shotgun metagenomes, bioRxiv 251462; doi: https://doi.org/10.1101/251462.

[40] Quince, C., Delmont, T.O., Raguideau, S., Alneberg, J., Darling, A.E., Collins, G., Eren, A.M. (2017). DESMAN: a new tool for de novo extraction of strains from metagenomes, Genome Biol. 18(1): 181

[41] Delmont, D.O., Quince, C., Shaiber, A., Esen, O.E., Lee, S.T.M., Rappé, M.S., McLellan, S.L., Lücker, S., Eren, A.M. Nitrogen-fixing populations of Planctomycetes and Proteobacteria are abundant in surface ocean metagenomes, Nature Microbiology 3: 804-813.

[42] Stewart, R.D., Auffret, M.D., Warr, A., Wiser, A.H., Press, M.O., Langford, K.W., Liachko, I., Snelling, T.J., Dewhurst, R.J., Walker, A.W., Roehe, R., Watson, M. (2018). Assembly of 913 microbial genomes from metagenomic sequencing of the cow rumen, Nature Communications, 9: 870

[43] Ji, P., Zhang, Y.M., Wang, J.F., Zhao, F.Q. (2017). MetaSort untangles metagenome assembly by reducing microbial community complexity. Nature Communications, $\mathbf{8}$, 14306.

[44] Burton, J.N., Liachko ,I., Dunham, M.J., Shendure, J. (2014). Species-level deconvolution of metagenome assemblies with $\mathrm{Hi}-\mathrm{C}-$ based contact probability maps. G3 (Bethesda), 4(7): 1339-46

[45] Bland, J.M., Altman, D.G. (1986). Statistical methods for assessing agreement between two methods of clinical measurement. Lancet 327 (8476): 307-10. 
bioRxiv preprint doi: https://doi.org/10.1101/465328; this version posted November 8, 2018. The copyright holder for this preprint (which was not certified by peer review) is the author/funder, who has granted bioRxiv a license to display the preprint in perpetuity. It is made available under aCC-BY-NC 4.0 International license.

565 [46] Lan, F., Demaree, B., Ahmed, N., Abate, A.R. (2017). Single-cell genome sequencing 566 at ultra-high-throughput with microfluidic droplet barcoding, Nat. Biotechnol. 35(7): $567 \quad 640-646$. 
Table 1: Summary statistics for long read data

\begin{tabular}{lc}
\hline \hline Measure & \\
\hline Number of reads & 115,383 \\
Number of reads after adapter trimming & 115,336 \\
Total length of sequenced reads & $539,410,340$ \\
Longest read (bp) & 112,729 \\
Shortest read (bp) & 3 \\
Number of reads $>500 \mathrm{bp}$ & 107,673 \\
Number of reads $>1000 \mathrm{bp}$ & 98,574 \\
Number of reads $>10 \mathrm{k} b \mathrm{p}$ & 6,324 \\
Number of reads $>100 \mathrm{k}$ bp & 1 \\
Number of reads $>1 \mathrm{M} \mathrm{bp}$ & 0 \\
Mean read length $(\mathrm{bp})$ & 4,677 \\
N50 (bp) & 6,502 \\
\hline
\end{tabular}


bioRxiv preprint doi: https://doi.org/10.1101/465328; this version posted November 8, 2018. The copyright holder for this preprint (which was not certified by peer review) is the author/funder, who has granted bioRxiv a license to display the preprint in perpetuity. It is made available under aCC-BY-NC 4.0 International license.

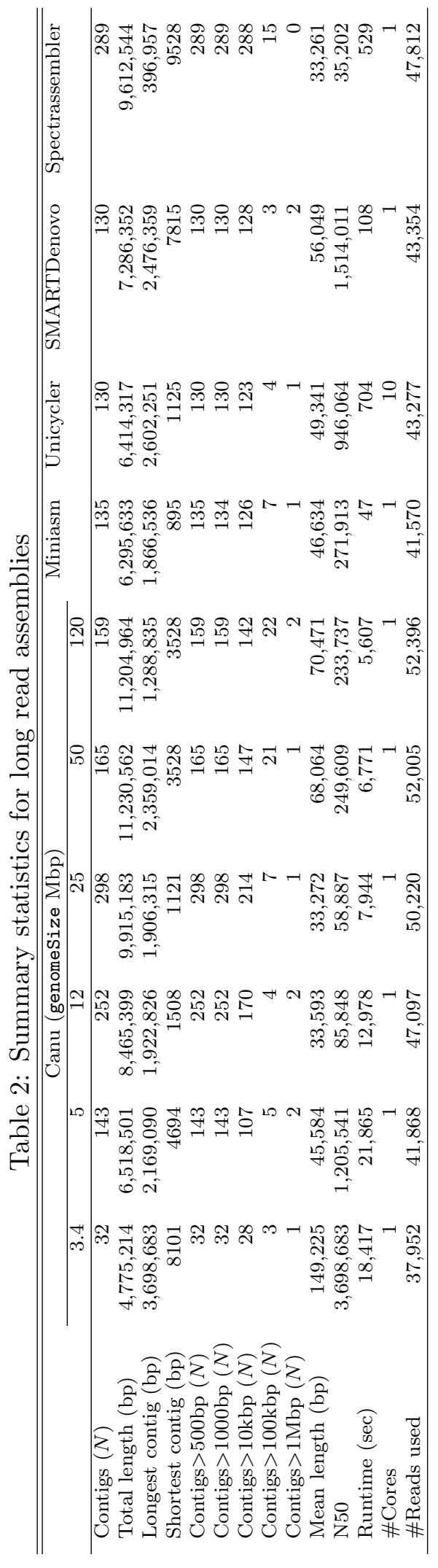


A.

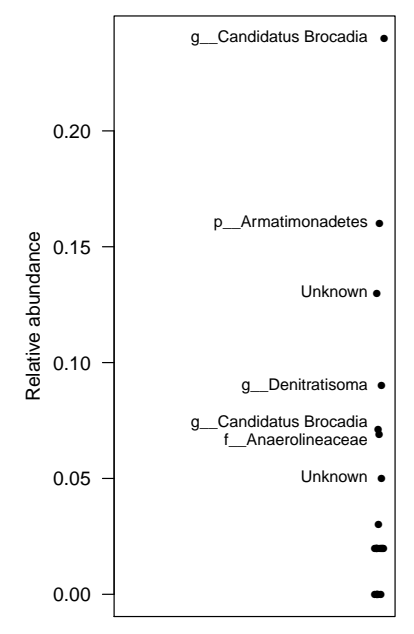

B.

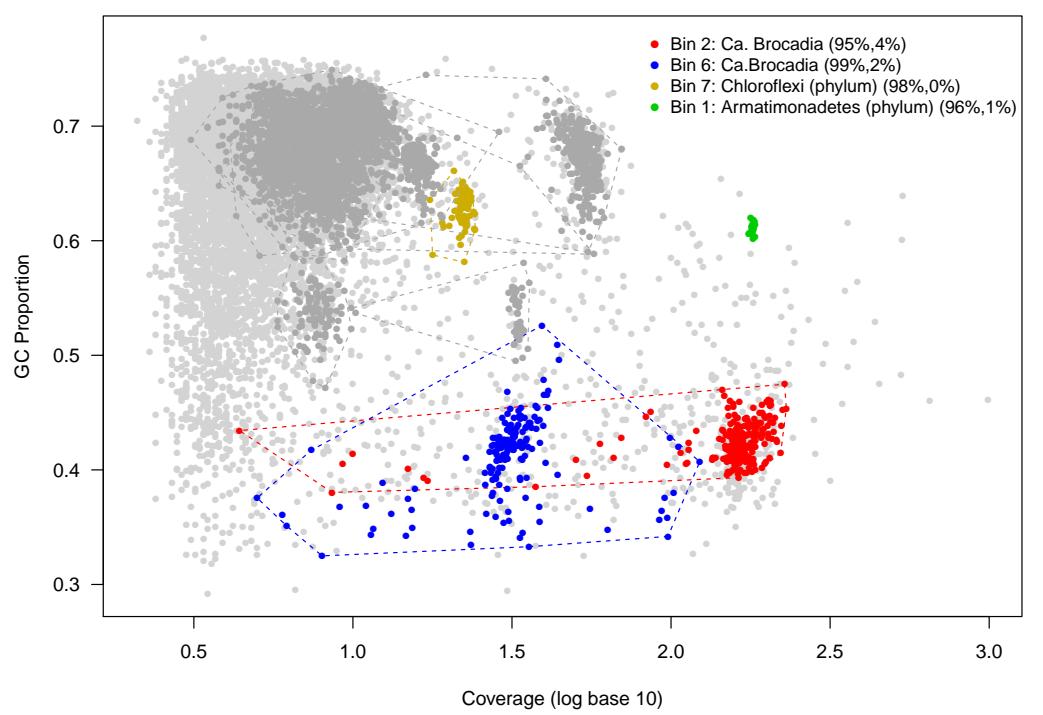

Figure 1: Analysis of short read sequencing data from AnAOB enrichment reactor community. A: RiboTagger analysis of short read data, showing taxon ranked by relative abundance. Unknown denotes cases in which RiboTagger could not assign any annotation to the corresponding ribotag sequence. A total of 23 taxa were detected, of which 7 had a relative abundance $\geq 0.05$ (named). A member of genus Candidatus Brocadia, a known AnAOB, was the most abundant community member, with a second member of this genus being present at a lower abundance. $B$ : Single-sample metgenome assembly of the short read sequence, constructed using Newbler v2.9. GC-proportion and log coverage are used to visualise contigs here. Contigs were binned using MetaBAT. A total of 16 bins were detected, of which 4 showed CheckM defined completeness $\geq 95 \%$ and contamination $\leq 5 \%$ (contigs shown in colours and described in legend: numbers in brackets show estimated contamination and completeness). Evidence supporting taxonomic assignments to bin is made on the basis of presence of full length, annotated 16S sequences, marker gene analysis in CheckM and ORF-level annotation statistics (see main text for details). 
bioRxiv preprint doi: https://doi.org/10.1101/465328; this version posted November 8, 2018. The copyright holder for this preprint (which was not certified by peer review) is the author/funder, who has granted bioRxiv a license to display the preprint in perpetuity. It is made available under aCC-BY-NC 4.0 International license.

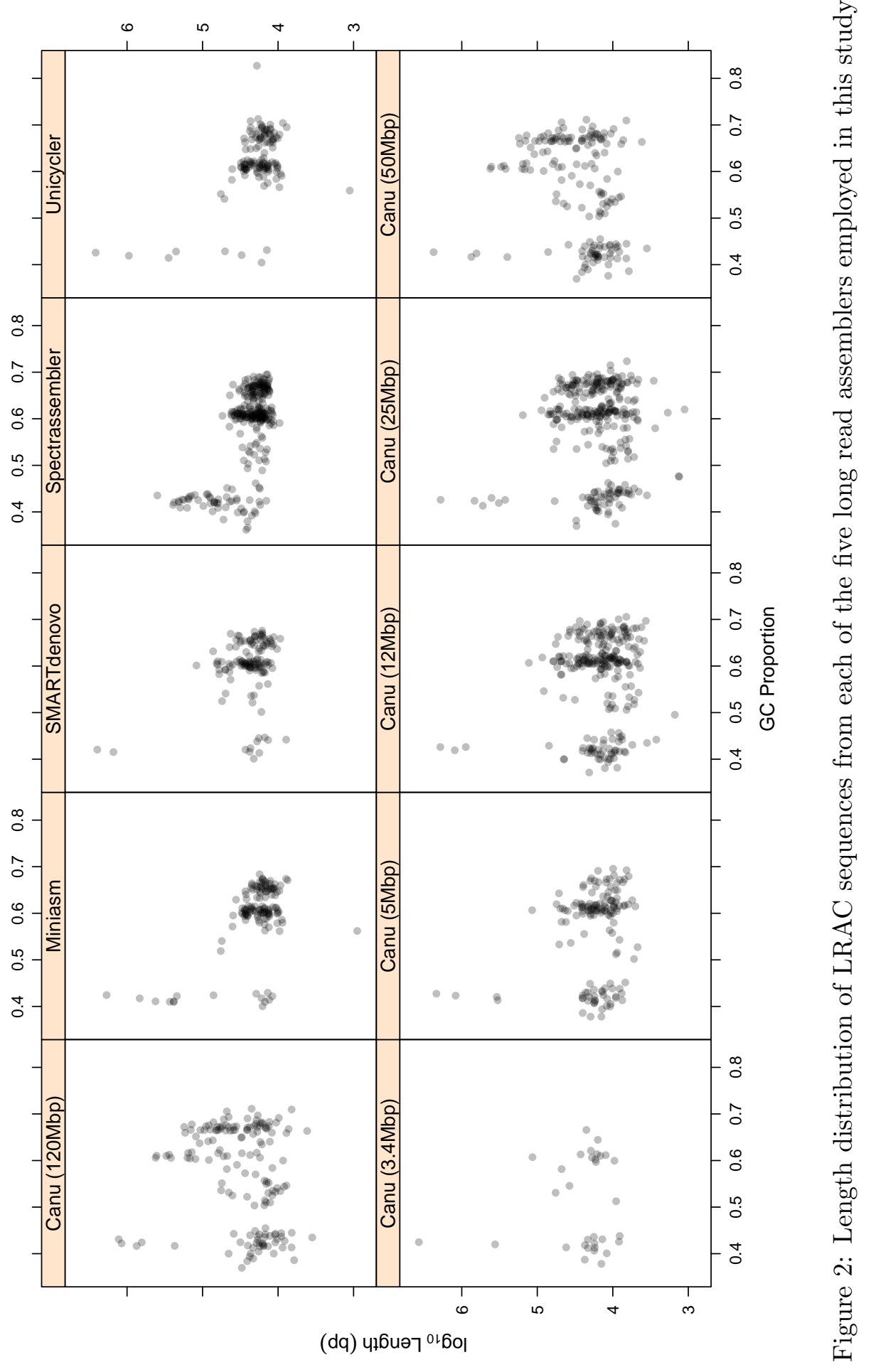



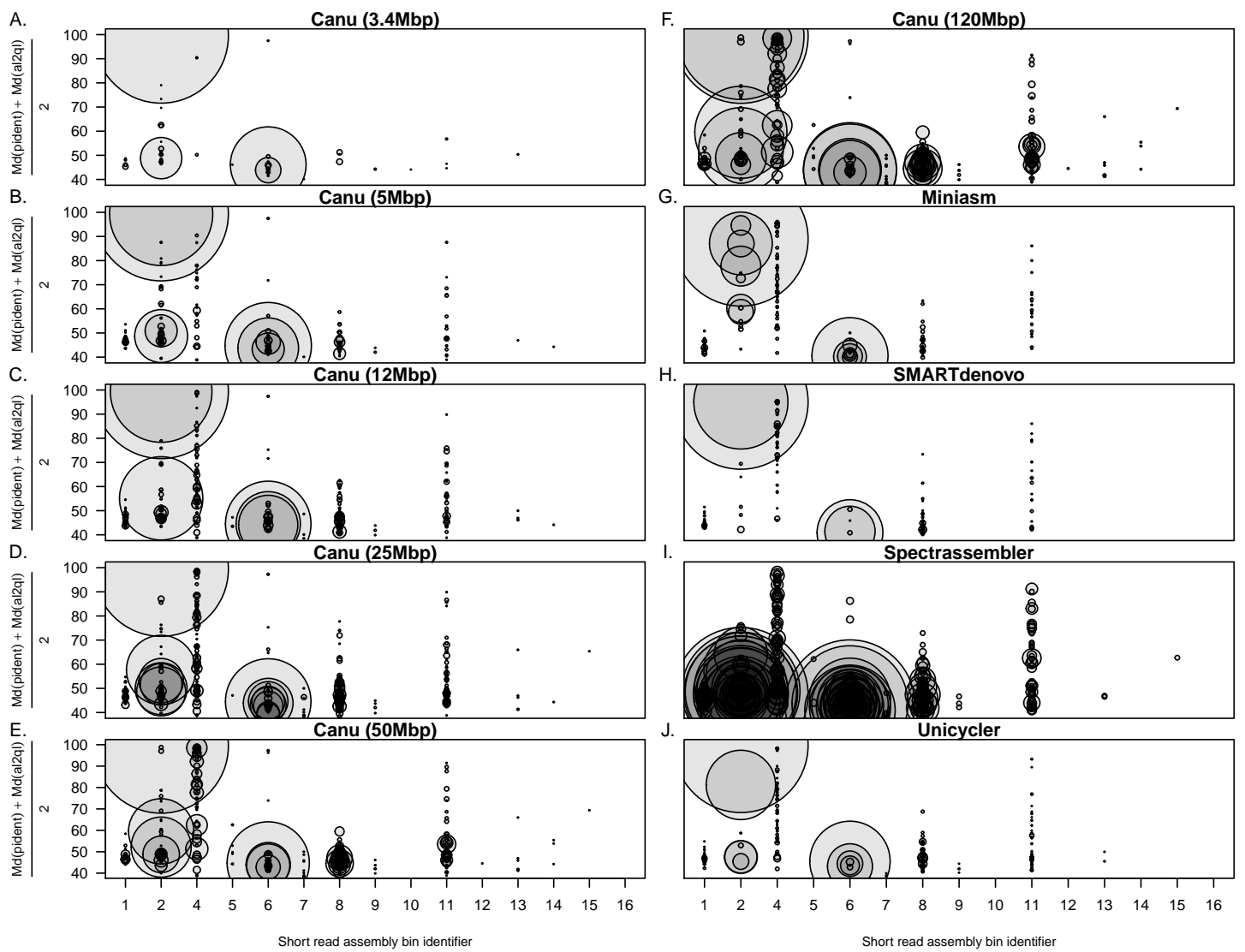

Figure 3: Summary of BLASTN alignment statistics between SRAC sequences and LRAC sequences categorised by bin membership of the SRAC sequences. In each panel, the horizontal axis lists SRAC bin identifiers (see Figure 1 and Table S1). Using BLASTN, SRAC sequences were treated as queries and LRAC sequences as the subject (see Methods: Comparative analysis of long and short read assemblies). Each data point corresponds an LRAC sequence with diameter proportional the mean of the median of the distribution of the percent identity (pident) and the median of the distribution of the alignment length to query (SRAC) length ( $a l 2 q l$; expressed as a percentage to be on the same scale as pident). Note that the LRAC sequences show a large number of highly aligned SRAC sequences that arising from the $\mathrm{Ca}$. Brocadia genome from bin 2, with a lesser degree of alignment evident from the second $C a$. Brocadia genome captured by SRAC sequence from bin 6 . 

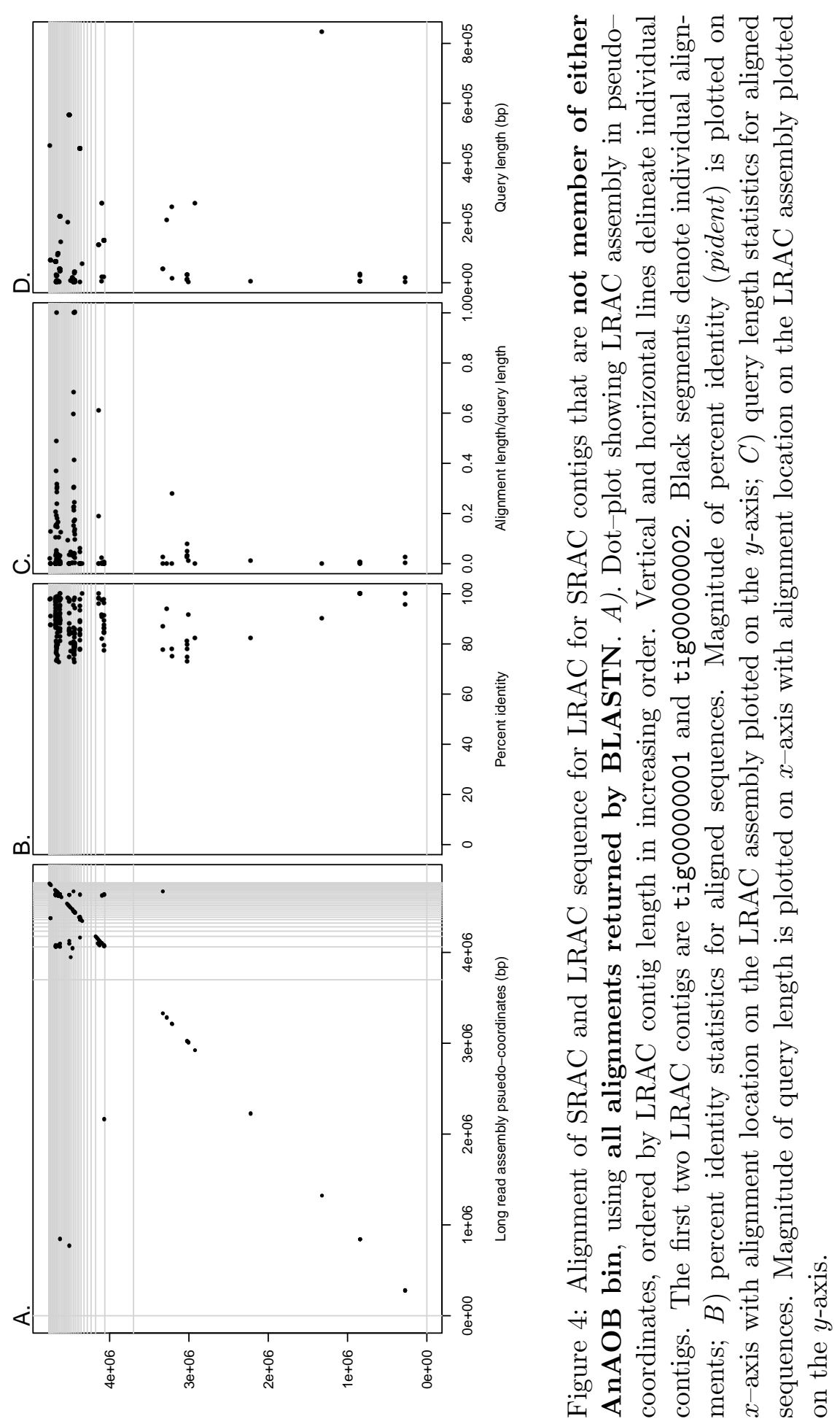

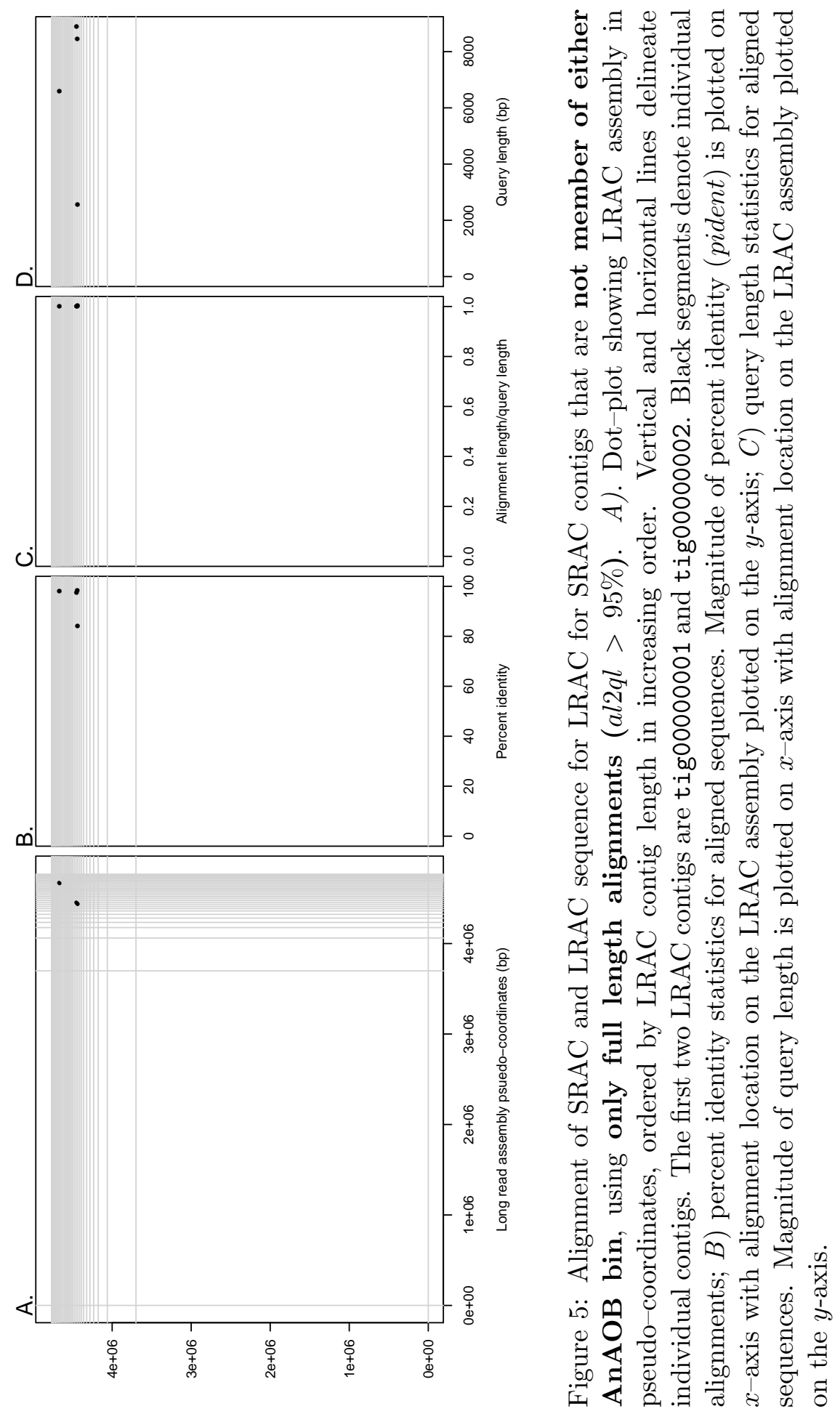

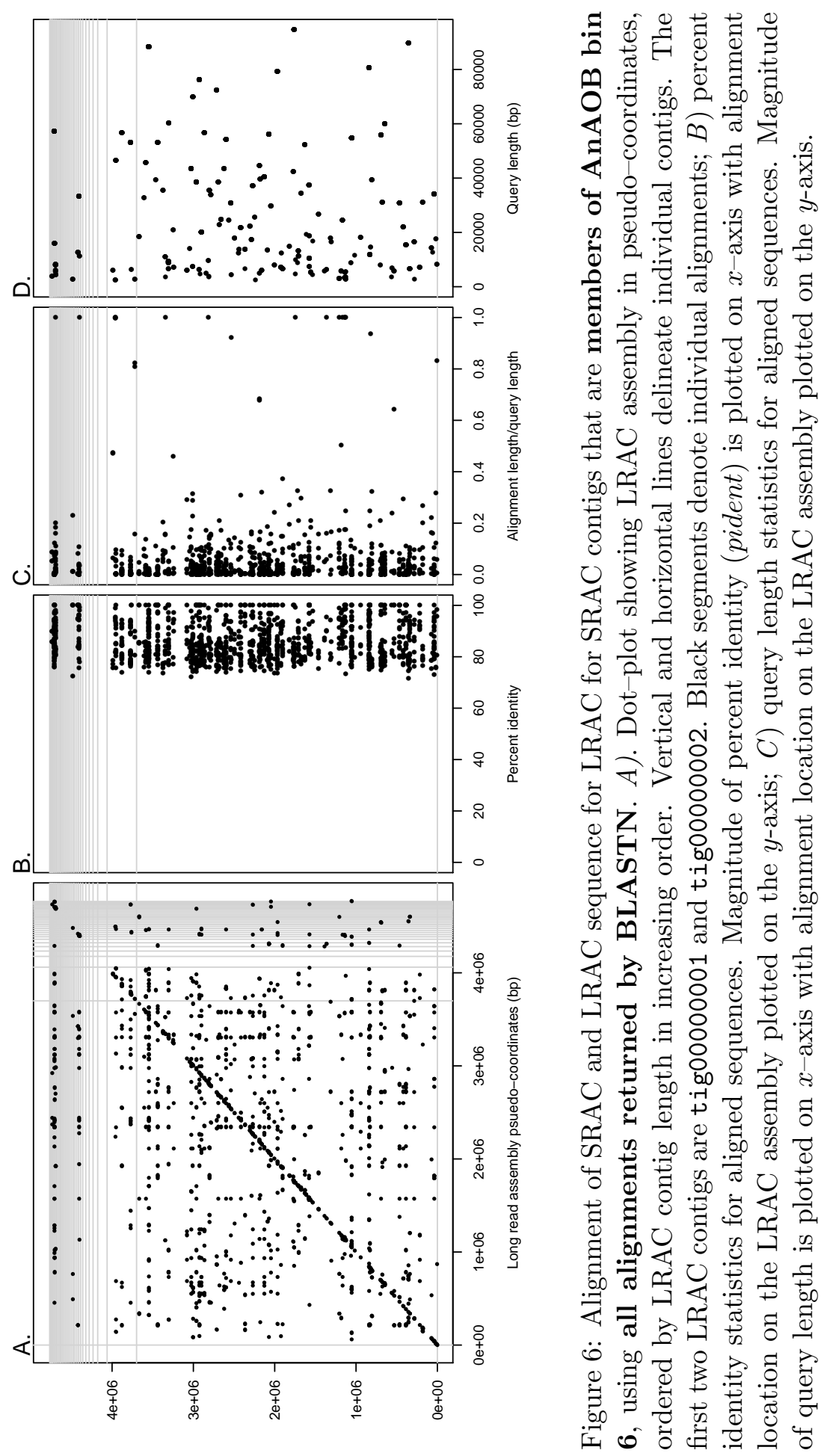


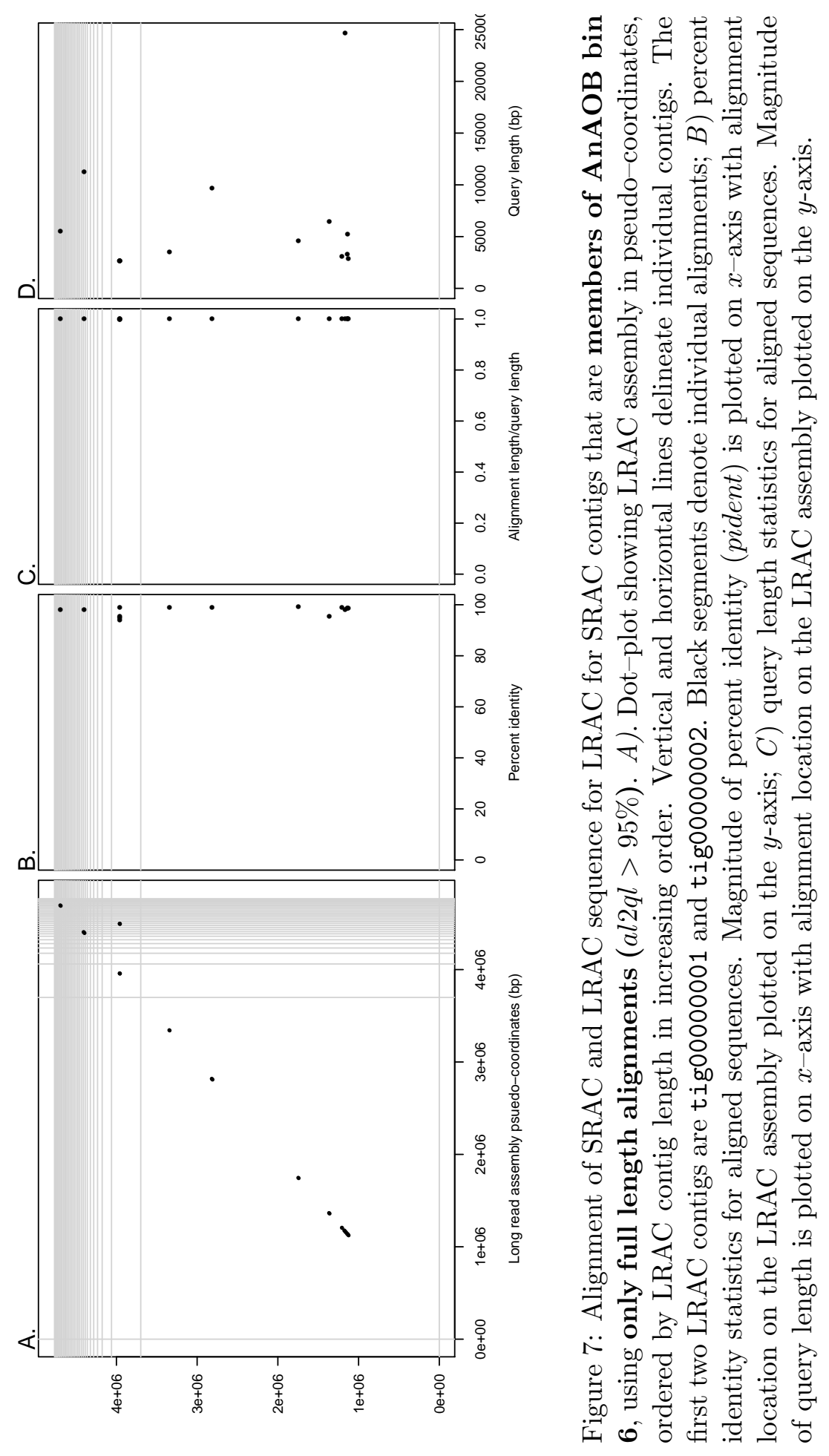


bioRxiv preprint doi: https://doi.org/10.1101/465328; this version posted November 8,2018 . The copyright holder for this preprint (which was not certified by peer review) is the author/funder, who has granted bioRxiv a license to display the preprint in perpetuity. It is made available under aCC-BY-NC 4.0 International license.

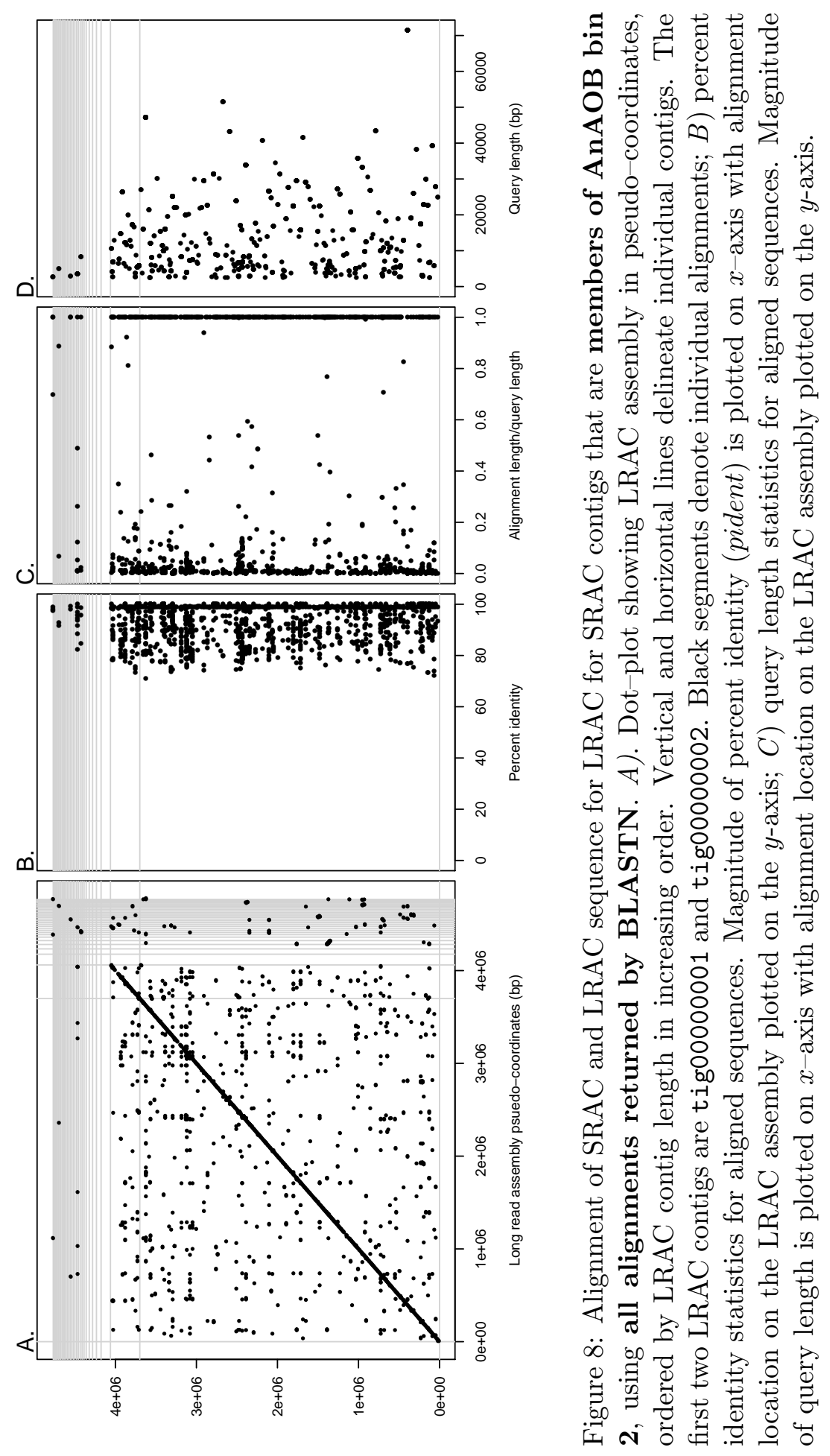




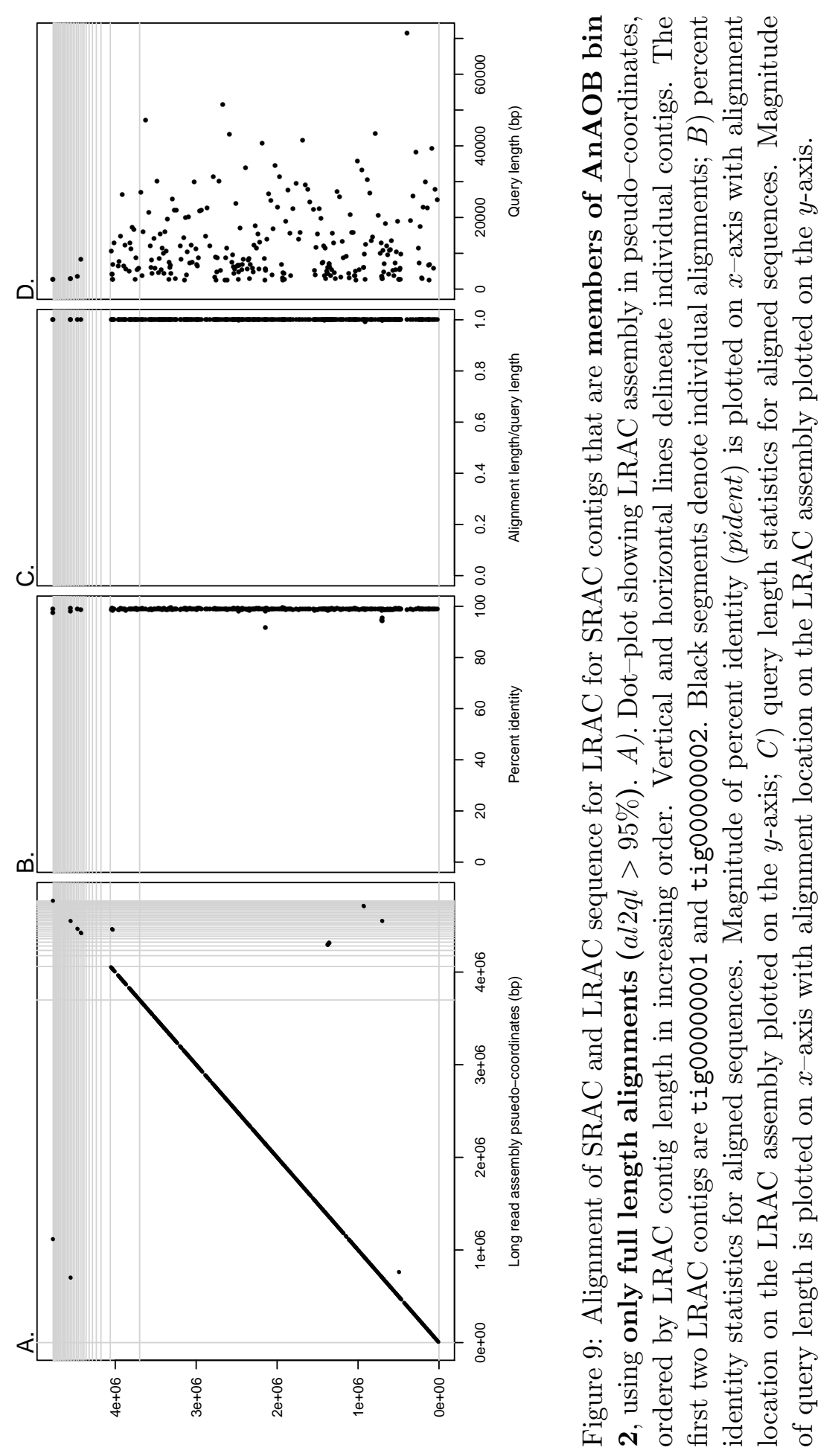



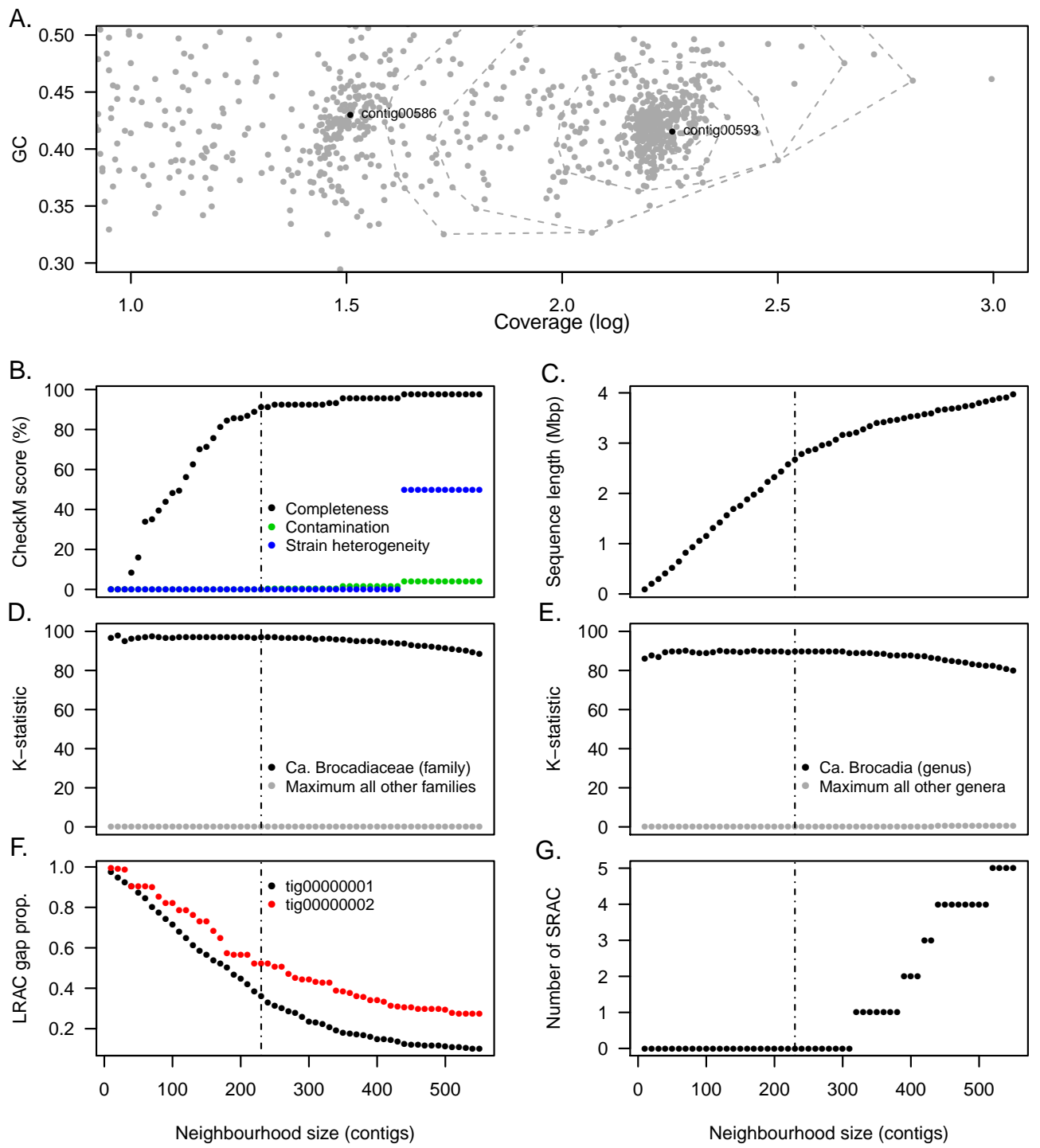

Figure 10: Re-analysis of AnAOB bin 2 using $k$-nearest neighbour contig sets (neighbourhoods) and alignments to LRAC sequence. A). Coverage $\left(\log _{10}\right)$-GC plane showing all contigs wih length $\geq 1 k b p$. Contig neighbourhoods (dashed convex hulls) are defined using the $k$-nearest neighbours of contig00593 which harbours a full length $16 \mathrm{~S}$ gene annotated to $C a$. Brocadia. In $A)-G$, the value of $k$ is plotted on the $x$-axis with the following statistics on the $y$-axis: $B$ ) CheckM genome quality statistics (completeness, contamination and strain heterogeneity); $C$ ) total sequence length (bp); D) ORF-level annotation $(K)$ statistics summarised at family level for $C a$. Brocadiaceae (black) and maximum observed over all other families (grey) E) ORF-level annotation $(K)$ statistics summarised at genus level for $C a$. Brocadia (black) and maximum observed over all other genera (grey); $F$ ) the proportion of LRAC sequence of tig00000001 and tig00000002 that contain no aligned SRAC sequence from bin $2 ; G$ ) the number of bin 2 SRAC sequenced that were unaligned to LRAC sequences. When calculating gaps in the alignments to LRAC sequence, we only considered full length SRAC aligned sequences (i.e. those with al2ql >0.95). 
Table S1: Summary statistics for the short read assembly

\begin{tabular}{lc}
\hline \hline Measure & \\
\hline Contigs $(N)$ & 38,988 \\
Total length (bp) & $75,821,912$ \\
Longest contig (bp) & 838,453 \\
Shortest contig (bp) & 500 \\
Contigs $>500$ bp $(N)$ & 38,910 \\
Contigs $>1000$ bp $(N)$ & 14,450 \\
Contigs $>10 \mathrm{k} \mathrm{bp}(N)$ & 813 \\
Contigs $>100 \mathrm{k}$ bp $(N)$ & 37 \\
Contigs $>1 \mathrm{M}$ bp $(N)$ & 0 \\
Mean length $(\mathrm{bp})$ & 1,945 \\
N50 & 4,145 \\
\hline
\end{tabular}




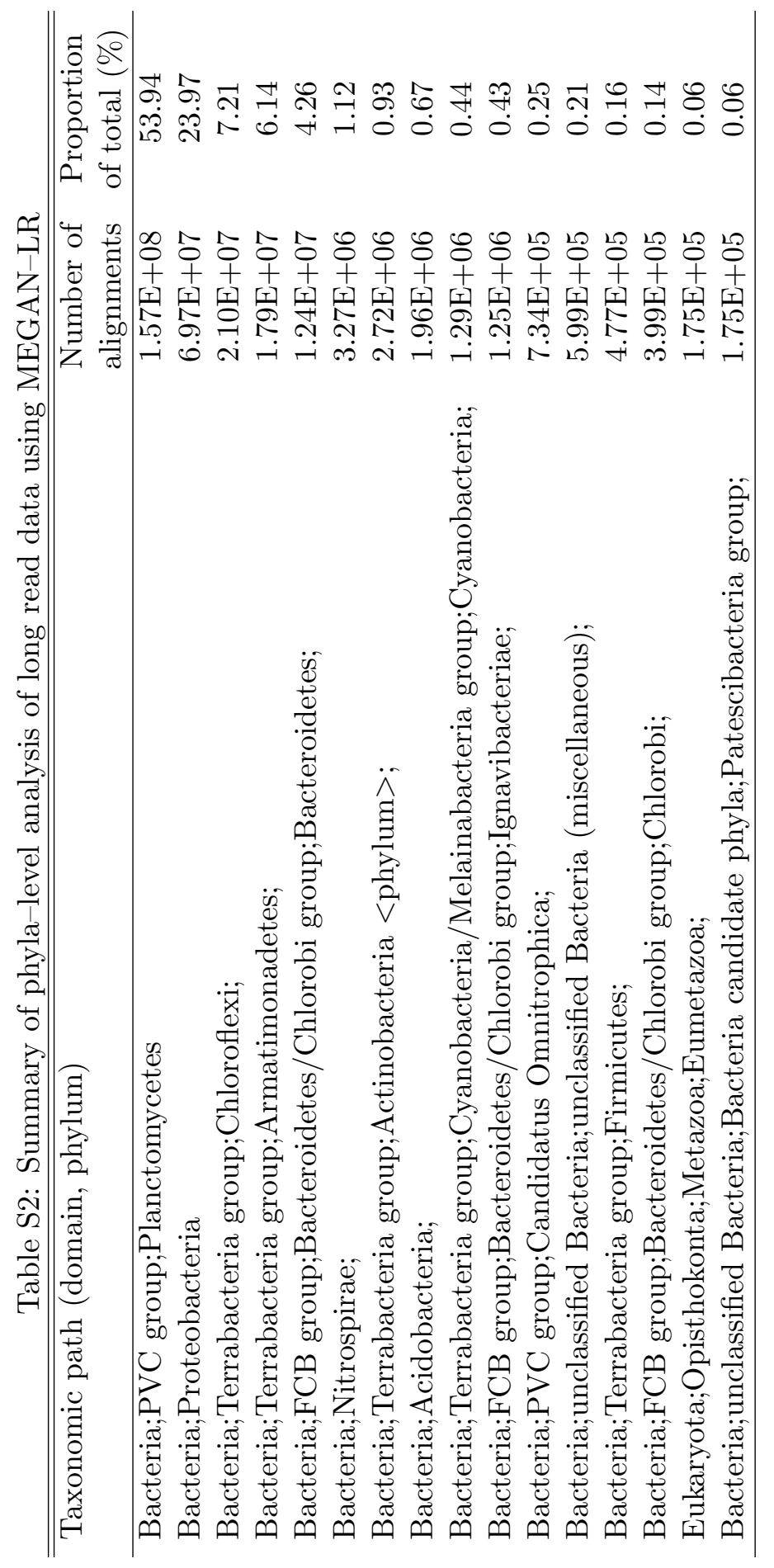




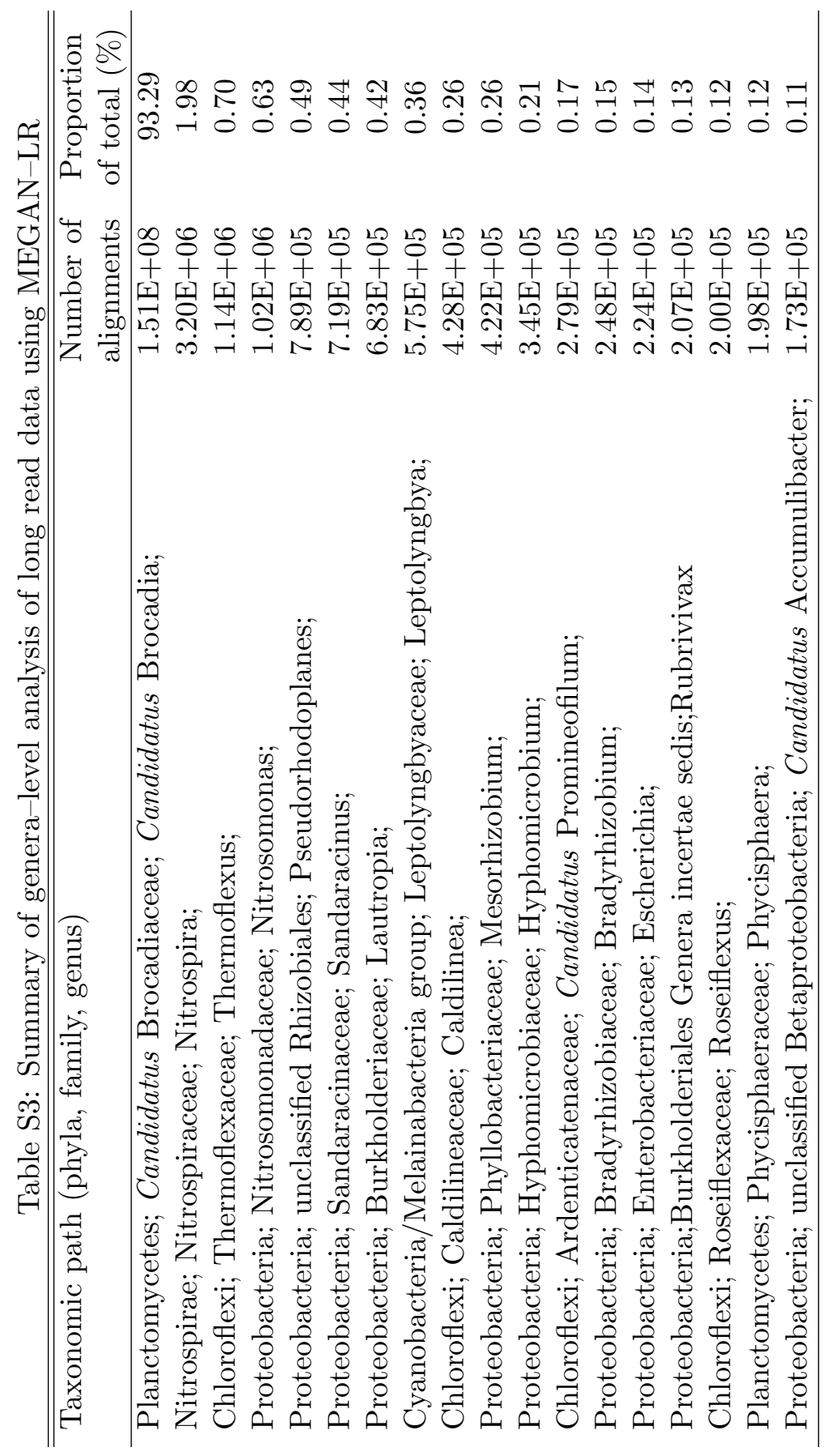




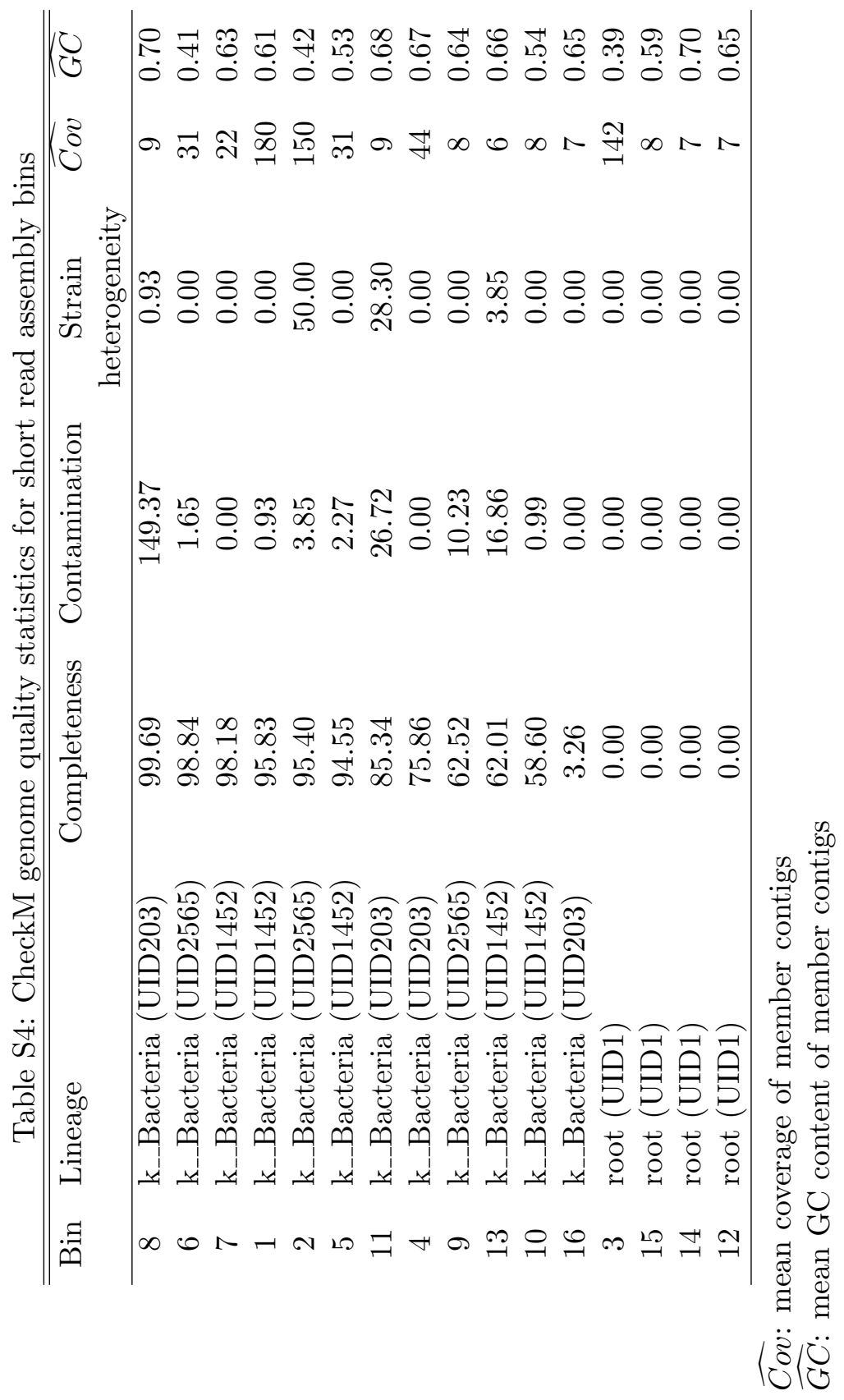




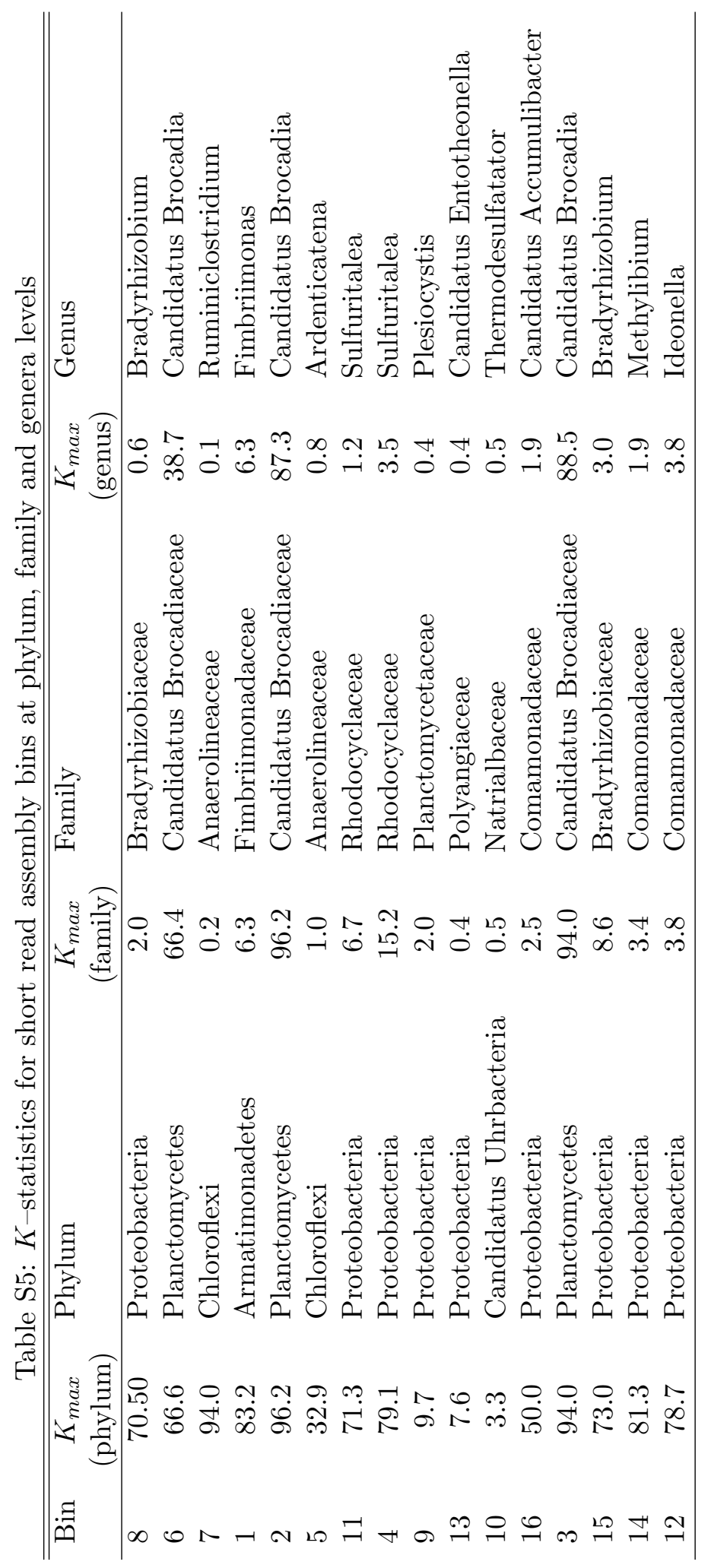


bioRxiv preprint doi: https://doi.org/10.1101/465328; this version posted November 8, 2018. The copyright holder for this preprint (which was not certified by peer review) is the author/funder, who has granted bioRxiv a license to display the preprint in perpetuity. It is made available under aCC-BY-NC 4.0 International license.
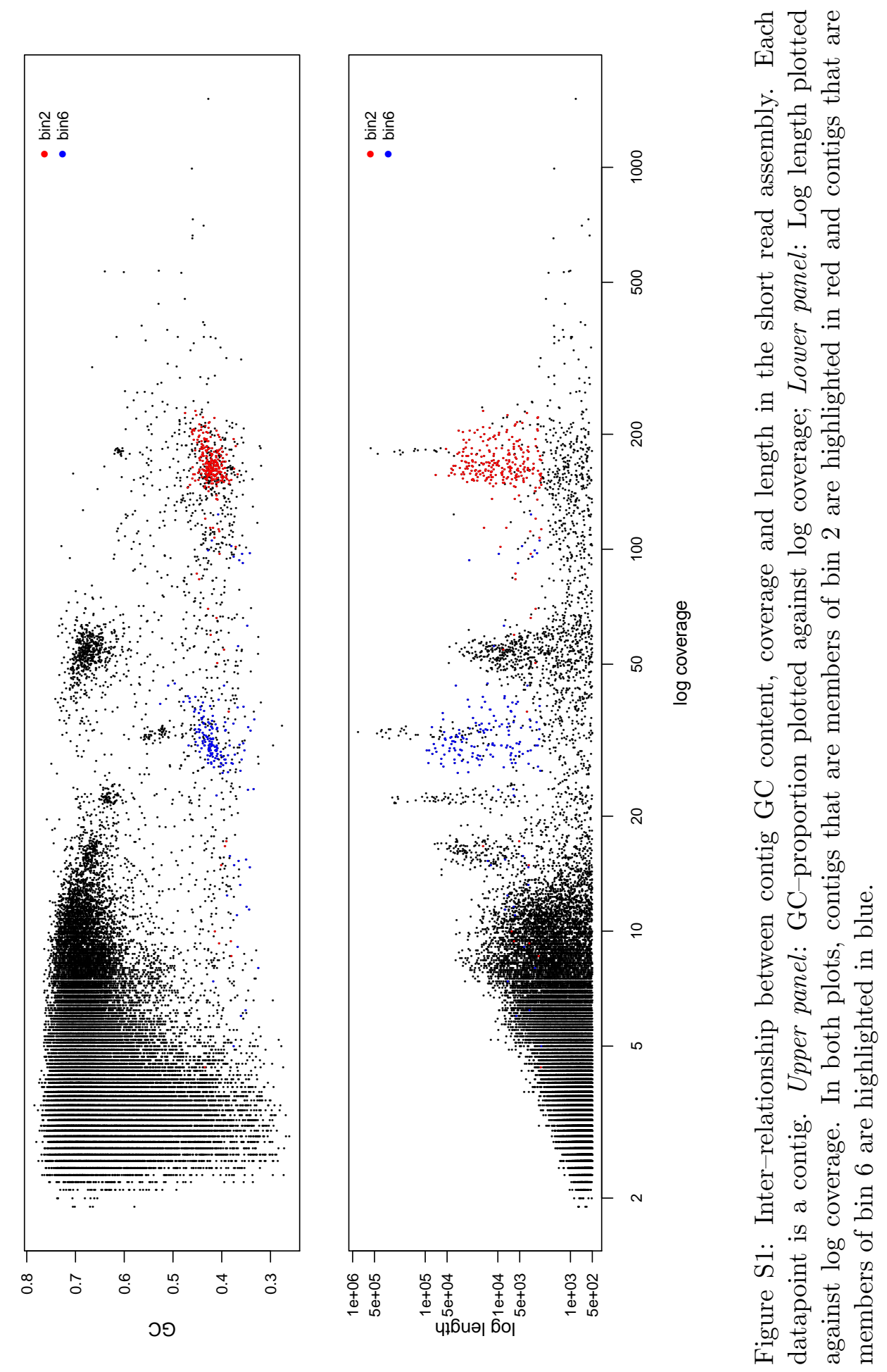
$\dot{\varphi}$
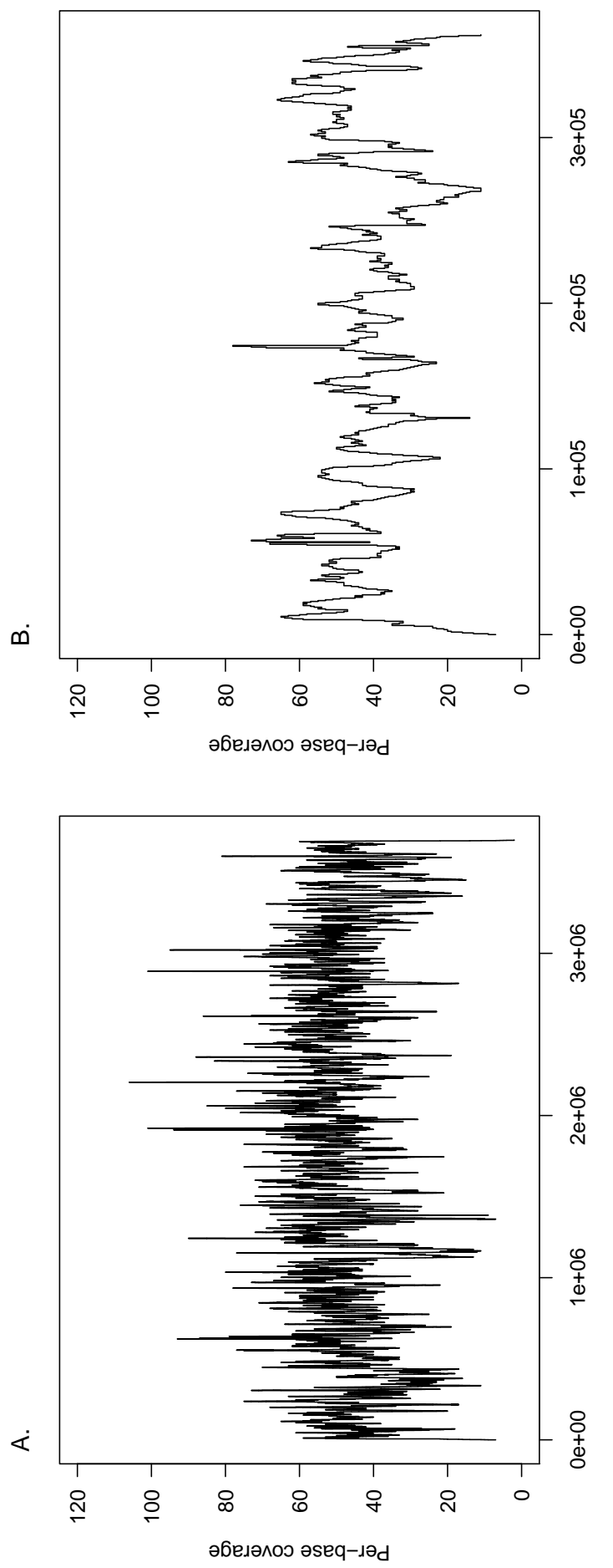

$4 \stackrel{0}{\square}$

ํํㅇ త్త

응

ఠิ

焉

$\ddot{\sim}$

록

శี

‥

웅 흐

응

응ㅎㅇ

bo

$\nabla$

\&.

包

常

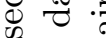

บ 800

$\checkmark$ త్ర

त्र

-11

co

0

ब0

ఫ)

合究

ซ్ల

¿ $\frac{1}{\dagger}$

$\stackrel{0}{2} \circ$

o

0

需

을

迥

ర

过

의

घ्व

ఫีత్తి

. O

i

is so

ㄴ.

50

I 